\title{
TORSORS OVER THE PUNCTURED AFFINE LINE
}

\author{
V. CHERNOUSOV, P. GILLE, AND A. PIANZOLA
}

\begin{abstract}
We classify $G$-torsors over the punctured affine line $\operatorname{Spec}\left(k\left[t^{ \pm}\right]\right)$ where $G$ is a reductive algebraic group defined over a field $k$ of good characteristic. Our classification is in terms of the Galois cohomology of the complete field $k((t))$ with values in $G$.

Keywords: Linear algebraic group, group scheme, torsor, punctured affine line, non-abelian cohomology. $M S C 2000$ 11E72, 14L30, 14E20.
\end{abstract}

\section{Contents}

1. Introduction

2. Statement of the two Main Theorems

3. Preliminary results 5

3.1. Notation and conventions 5

3.2. Generalities on torsors 6

3.3. Twisting 7

3.4. Reducibility and isotropy 11

3.5. Patching group schemes 12

3.6. Weil restriction considerations 15

4. Torsors over the affine line 19

5. Torsors over the punctured affine line 22

5.1. Cohomological exponent: Good and bad primes 23

5.2. Existence of maximal tori 25

5.3. Reformulation of Theorem $2.4 \quad 26$

5.4. Reduction of structure group to $N_{G}(T) \quad 28$

5.5. Proof of surjectivity 30

5.6. Proof of Injectivity 31

6. Appendix: The absolutely reduced subscheme attached to a scheme 38

$\begin{array}{ll}\text { References } & 42\end{array}$

V. Chernousov was partially supported by the Canada Research Chairs Program and an NSERC research grant.

A. Pianzola wishes to thank NSERC and CONICET for their continuous support. 


\section{INTRODUCTION}

Let $G$ be a reductive group over a field $k$. The main objective of our work is to give a description of the isomorphism classes of $G$-torsors over the punctured affine line $\mathbb{A}_{k}^{\times}=\operatorname{Spec}\left(k\left[t^{ \pm 1}\right]\right)$ in terms of the Galois cohomology of the complete field $k((t))$ with values in $G$. The relevance of this characterization is that the latter cohomology set $H^{1}(k((t)), G)$ can be computed using the methods developed by Bruhat and Tits [BT3].

Many interesting objects defined over a rational function field $k(t)$ can usually be described, or at least be partially understood, through their residues. Examples of this type of behaviour are the famous exact sequences related to Milnor $K$-theory, Brauer groups and Witt groups. The exact sequence for Milnor's $K$-groups [Mn, $\S 2]$ is given by

$$
0 \rightarrow K_{n}^{M}(k) \rightarrow K_{M}^{n}(k(t)) \stackrel{\partial}{\longrightarrow} \oplus_{p} K_{n-1}^{M}(k(p)) \rightarrow 0
$$

where $p$ runs over the closed points of the affine line. Similarly, if $k$ is perfect, we have Faddeev's exact sequence [GS, §6.4]

$$
0 \rightarrow \operatorname{Br}(k) \rightarrow \operatorname{Br}(k(t)) \stackrel{\partial}{\longrightarrow} \oplus_{p} H^{1}(k(p), \mathbb{Q} / \mathbb{Z}) \rightarrow 0
$$

and the exact sequence for Witt groups

$$
0 \rightarrow W(k) \rightarrow W(k(t)) \stackrel{\partial}{\longrightarrow} \oplus_{p} W(k(p)) \rightarrow 0
$$

in odd characteristic [Mn, §5].

Brauer groups and Witt groups are closely related to projective linear groups and orthogonal groups respectively. One is thus lead to consider the possibility that there may exist analogues of the last two of the above exact sequences for $G$-torsors over $k(t)$. To study this problem the (traditional) local-global approach leads us to look in detail at the natural map

$$
a=\prod a_{p}: H^{1}(k(t), G) \longrightarrow \prod_{p \in \mathbb{P}_{k}^{1}} H^{1}\left(k(t)_{p}, G\right)
$$

where $p$ runs through the set of closed points of the projective line $\mathbb{P}_{k}^{1}, k(t)_{p}$ is the completion of $k(t)$ with respect to the discrete valuation $v_{p}$ associated to $p$, and $a_{p}: H^{1}(k(t), G) \rightarrow H^{1}\left(k(t)_{p}, G\right)$ is the natural restriction map. Note that, unlike the case of Brauer groups and Witt groups, the set of isomorphism classes of $G$-torsors over $k(t)$ is not a group. It is therefore unrealistic to expect that a unique short exact sequence with $H^{1}(k(t), G)$ as middle term could describe all $G$-torsors over $k(t)$. The only reasonable hope is to try to describe the fibers of the map $a$ in more or less acceptable terms. This leads to infinitely many exact sequences instead of just one, as we now explain.

We say that an element $x$ of $H^{1}(k(t), G)$ is unramified at $p$ if $a_{p}(x)$ is in the image of the natural map $H^{1}\left(\widehat{O}_{p}, G\right) \rightarrow H^{1}\left(k(t)_{p}, G\right)$, where $\widehat{O}_{p}$ is the ring of integers of $k(t)_{p}$. Otherwise we say that $x$ is ramified at at $p$. In the latter case, and if $G / k$ is quasi-split, using Bruhat-Tits theory we can 
associate to $x$ an element $x_{p}$ in $H^{1}\left(k(p), H_{p}\right)$ where $k(p)$ is the residue of $k(t)_{p}$, and $H_{p}$ is a proper subgroup of the $k(p)$-group $G_{k(p)}$ which depends on $x$. It seems natural to call $x_{p}$ the residue of $x$ at $p$ and call the finite set $S=\left\{p_{1}, \ldots, p_{n}\right\}$ where $x$ is ramified the ramification locus of $x$. By Bruhat-Tits theory $H^{1}\left(k(t)_{p}, G\right)$ is the disjoint union of "anisotropic parts" of $H^{1}\left(k(p), H_{p}\right)$, namely of those classes that arise from $[\xi] \in H^{1}\left(k(p), H_{p}\right)$ for which the twisted group ${ }_{\xi} H_{p}$ is anisotropic. This indicates that we could get a satisfactory description of $H^{1}(k(t), G)$ in terms of sets $H^{1}\left(k(p), H_{p}\right)$ if we knew the fibers of the map

$$
H^{1}(k(t), G)_{S} \longrightarrow \prod_{p \in S} H^{1}\left(k(t)_{p}, G\right)
$$

where $H^{1}(k(t), G)_{S}=a^{-1}\left(\prod_{p \in S} H^{1}\left(k(t)_{p}, G\right) \times \prod_{p \notin S} H^{1}\left(\widehat{O}_{p}, G\right)\right)$.

Note that $H^{1}(k(t), G)_{S}$ is the image of the natural mapping

$$
H^{1}(U, G) \rightarrow H^{1}(k(t), G)
$$

where $U=\mathbb{P}_{k}^{1} \backslash\left\{p_{1}, \ldots, p_{n}\right\}$ (see [H1]). Thus, out of necessity, we are forced to study the image of this map and its fibers.

Assume that characteristic of $k$ is good (see $\S 5.1$ for details). If $S=\emptyset$, then $H^{1}(k(t), G)_{S}=H^{1}(k, G)$ and the fibers of (1.2) are well understood [G1, §I.2]. If $S=\{\infty\}$ then by a theorem of Raghunathan-Ramanathan (see below) the natural maps $H^{1}(k, G) \rightarrow H^{1}\left(\mathbb{A}_{k}^{1}, G\right)$ is bijective and (1.2) is injective. In our paper we consider the next case, namely when $S$ consists of two points $S=\{0, \infty\}$. It turns out that the map (1.2) is again injective, and we can describe its image in terms of local $G$-torsors.

Our work is unequivocally motivated by that of Raghunathan and Ramanathan $[\mathrm{RR}]$, where the case of the affine line $\mathbb{A}_{k}^{1}=\operatorname{Spec}(k[t])$ is considered. Even though the overlap between the two works is at times evident (extending group schemes to $\mathbb{P}_{k}^{1}$, the use Weil restrictions to address representability questions...), there are also substantial differences, notably the use of some key results from the theory of multiplicative group schemes developed in [SGA3], as well as techniques pertaining to fundamental domains in buildings due to Soulé (for the affine line) and to Abramenko (in the case of the punctured line). These methods allow us to establish-by different means than those used in $[R R]$-the existence of maximal tori on certain reductive group schemes. This is one of the crucial points within the main proof. Since our methods work equally well both for the affine and the punctured affine line, we have decided to include a (short) section with a proof of Raghunathan and Ramanathan's original result.

One should observe that the nature of $G$-torsors over $\mathbb{A}_{k}^{1}$ and $\mathbb{A}_{k}^{\times}$are quite different, mostly owing to the fact that, unlike $\mathbb{A} \frac{1}{k}$, the scheme $\mathbb{A}_{\frac{x}{k}}$ is not simply connected (in the algebraic sense). In good characteristic for 
example, $G$-torsors over the affine line are always constant, but this need not be the case for $G$-torsors over the punctured line.

$$
* * *
$$

The main body of the paper is divided into four parts. We begin by giving the statement of the two main theorems. This is followed by a section on "Preliminary results" that provides the conventions, notation and terminology that are used throughout the paper, as well as some general results (some of independent interest) that are used in the proofs of the two main theorems. The proofs of the main theorems themselves are the content of the last two sections. An Appendix with a technical observation about absolutely reduced algebraic groups is included at the end.

\section{Statement of the two Main Theorems}

Let $X$ be a scheme, and $G$ a group scheme over $X$. For any scheme $Y$ over $X$ we denote the $Y$-group scheme $G \times_{X} Y$ by $G_{Y}$. Recall that a torsor over $X$ under $G$ is a scheme $E$ over $X$ equipped with a right action of $G$ for which there exists a faithfully flat morphism $Y \rightarrow X$, locally of finite presentation, such that $E \times_{X} Y \simeq G \times_{X} Y=G_{Y}$, where $G_{Y}$ acts on itself by right translation. The set of isomorphisms classes of $X$-torsors under $G$ is denoted by $H^{1}(X, G)$.

Let $G$ be a linear algebraic over a field $k$ and $X$ a $k$-scheme. The structural morphism $X \rightarrow \operatorname{Spec}(k)$ yields a natural map

$$
\eta: H^{1}(k, G) \longrightarrow H^{1}(X, G):=H^{1}\left(X, G_{X}\right) .
$$

Let $E$ be a torsor over $X$ under $G_{X}$. We say that $E$ is constant if its isomorphism class belong to the image of the map $\eta$, and that $E$ is geometrically separably trivial if $E$ becomes trivial after the base change $X \times_{k} k_{s} \rightarrow X$.

2.1. Theorem. [Raghunathan-Ramanathan] Let $G$ be a linear algebraic $k$ group whose connected component of the identity is reductive. ${ }^{1}$ Every torsor over the affine line $\mathbb{A}_{k}^{1}$ under $G$ which is geometrically separably trivial is constant.

2.2. Remark. According to the bijection (3.3) described in Remark 3.2, the isomorphism classes of geometrically separably trivial $\mathbb{A}_{k}^{1}$-torsors under $G$ are classified by $H^{1}\left(\operatorname{Gal}\left(k_{s} / k\right), G\left(\mathbb{A}_{k_{s}}^{1}\right)\right)$. The natural map $\eta: H^{1}(k, G) \longrightarrow$ $H^{1}\left(\mathbb{A}_{k}^{1}, G\right)$ corresponds to the natural map

$$
H^{1}\left(\operatorname{Gal}\left(k_{s} / k\right), G\left(k_{s}\right)\right) \longrightarrow H^{1}\left(\operatorname{Gal}\left(k_{s} / k\right), G\left(\mathbb{A}_{k_{s}}^{1}\right)\right)
$$

arising from the inclusion $G\left(k_{s}\right) \subset G\left(\mathbb{A}_{k_{s}}^{1}\right)$. The map (2.3) is injective because of the existence of rational points on $\mathbb{A}_{k}^{1}$. Theorem 2.1 is thus equivalent to (2.3) being bijective. In good characteristic, it is known that every $G$-torsor over $\mathbb{A}_{k}^{1}$ is geometrically separably trivial, hence constant. In bad

\footnotetext{
${ }^{1}$ In $[R R]$ the group $G$ is assumed to be connected and reductive. The generalization we give here is not difficult to obtain from the reductive case. See $\S 4.3$ below.
} 
characteristic, this is in general not true anymore, even for a semisimple simply connected group (see $[\mathrm{G} 3, \S 2.4]$ for details).

The second part of our work gives a description of torsors over the punctured line $\mathbb{A}_{k}^{\times}=\operatorname{Spec}\left(k\left[t^{ \pm 1}\right]\right)$. It is not true that in this case geometrically separably trivial torsors are constant, even when $G$ is semisimple. The correct parametrization is obtained by looking at the base change corresponding to the completion of the generic fiber. The inclusion $k\left[t^{ \pm 1}\right] \subset k((t))$ yields a natural map $H^{1}\left(\mathbb{A}_{k}^{\times}, G\right) \rightarrow H^{1}(k((t)), G)$. Our result shows that, under a certain assumption on the characteristic of the base field, this map is bijective.

2.4. Theorem. Let $G$ be a (connected) reductive algebraic group over $k$. Assume that the characteristic of $k$ is good for $G$ (see 5.1). Then the natural map $H^{1}\left(\mathbb{A}_{k}^{\times}, G\right) \rightarrow H^{1}(k((t)), G)$ is bijective. In other words, the set of isomorphism classes of $\mathbb{A}_{k}^{\times}$-torsors under $G$ is parametrized by the usual Galois cohomology of $G$ over the complete field $k((t)){ }^{2}$

\section{Preliminary Results}

This section contains the conventions, notation and terminology that are used throughout the paper. It also contains results that are common to the proofs of the main theorems.

3.1. Notation and conventions. Throughout $k$ denotes a field, $\bar{k}$ an algebraic closure of $k$, and $k_{s}$ the separable closure of $k$ in $\bar{k}$. Given a scheme $X$ over $k$ and a field extension $k^{\prime} / k$ we set

$$
X_{k^{\prime}}:=X \times_{k} k^{\prime}
$$

For convenience we denote $X_{k_{s}}$ simply by $X_{s}$.

Most of our work is related to group schemes over a given base scheme $X$. For convenience we will sometime refer to these simply as $X$-groups. If $X=$ $\operatorname{Spec}(R)$ we use the terminology $X$-group and $R$-group indistinctively. We recall that given an $X$-group $G$ and a scheme $Y$ over $X$ the $Y$-group $G \times_{X} Y$ is denoted by $G_{Y}$. That " $Y$ is a scheme over $X$ " is at times abbreviated by simply writing $Y / X$.

By a reductive $X$-group we will understand a reductive group scheme over $X$ in the sense of [SGA3]. Accordingly, a reductive $k$-group is a connected reductive group defined over $k$ in the sense of Borel [Bor]. If $G$ is a reductive $X$-group, then the concept of maximal tori, parabolic subgroup, Levi subgroup... of $G$ is again the one given by [SGA3].

Let $F$ be a field extension of $k$. The additive and multiplicative groups over $F$ will be denoted by $\mathrm{G}_{a, F}$ and $\mathrm{G}_{m, F}$ respectively, or simply by $\mathrm{G}_{a}$ and $\mathrm{G}_{m}$ when $F=k$. The separable closure of $F$, which will always be taken in some algebraic closure $\bar{F}$ of $F$ that contains $\bar{k}$, will be denoted by $F_{\text {sep }}$.

\footnotetext{
${ }^{2}$ The set $H^{1}(k((t)), G)$ is well understood from the work of Bruhat-Tits [BT3].
} 
Thus $k_{\text {sep }}=k_{s}$, but in general one should not confuse $F_{\text {sep }}$ with $F_{s}$ (which by definition equals $F \otimes_{k} k_{s}$, and plays no role in our work).

Given a field extension $F$ of $k$ and a torus $T$ over $F$, there is a natural action of the Galois group $\operatorname{Gal}\left(F_{\text {sep }} / F\right)$ on the (abstract) group

$\operatorname{Hom}_{F_{\text {sep }}-\operatorname{grp}}\left(\mathrm{G}_{m, F_{\text {sep }}}, T_{F_{\text {sep }}}\right)$. We call the resulting Galois module the group of cocharacters of $T$, and denote it by $X(T)_{*}$.

3.2. Generalities on torsors. Let $X$ be a scheme, and $G$ a group scheme over $X$. As it is customary, for any scheme $Y$ over $X$ we denote by $p_{i}, i=1,2$, the corresponding projection $Y \times_{X} Y \rightarrow Y$ on the $i$-th component and by $p_{i j}, i, j=1,2,3$, the projection $Y \times_{X} Y \times_{X} Y \rightarrow Y \times_{X} Y$ on the $i j$-th component. These projections naturally induce group homomorphisms

$$
G(Y) \rightarrow G\left(Y \times_{X} Y\right) \text { and } G\left(Y \times_{X} Y\right) \rightarrow G\left(Y \times_{X} Y \times_{X} Y\right)
$$

which we still denote by $p_{i}$ and $p_{i j}$ (instead of the more usual $p_{i}^{*}$ and $p_{i j}^{*}$ notation).

Assume now that $Y / X$ is an fppf cover of $X$, that is $Y \rightarrow X$ is a scheme morphism which is faithfully flat and locally of finite presentation ([SGA3], IV.6.4). ${ }^{3}$ For such a covering $Y \rightarrow X$, we define the corresponding set of cocycles

$$
\check{Z}^{1}(Y / X, G):=\left\{g \in G\left(Y \times_{X} Y\right) \mid p_{23}(g) p_{12}(g)=p_{13}(g)\right\}
$$

and non-abelian cohomology

$$
\check{H}^{1}(Y / X, G):=\check{Z}^{1}(Y / X, G) / G(Y),
$$

where $G(Y)$ acts on $\check{Z}^{1}(Y / X, G)$ by $g \cdot z=p_{2}(g) z p_{1}(g)^{-1}$ (see [H1], $\S 1.3$ and $[\mathrm{M}]$ for details). For convenience we will at times use the notation $\check{Z}^{1}(X, G)$ to denote the totality of cocycles $\check{Z}^{1}(Y / X, G)$ with $Y$ as above variable (within a given range, which would always be clear from the context, so as to avoid set-theoretical problems). We now define

$$
\check{H}^{1}(X, G):=\underset{Y}{\lim _{Y}} \check{H}^{1}(Y / X, G),
$$

where the limit is taken over all (equivalence classes of) fppf covers $Y \rightarrow X$.

3.1. Remark. One defines in an exact analogous fashion $\check{H}^{1}(X, G)$ whenever $G$ is a sheaf of groups for the fppf topology on $X$.

Recall that the set of isomorphisms classes of $X$-torsors under $G$ is denoted by $H^{1}(X, G)$. Thus $H^{1}(X, G)$ is a pointed set; its distinguished class, which we denote by 1 , is the class of the trivial torsor, namely the scheme $G$ acting on itself by right translation. The subset of $H^{1}(X, G)$ corresponding to torsors which are trivialized by a given (arbitrary) base change $X^{\prime} \rightarrow X$ is denoted by $H^{1}\left(X^{\prime} / X, G\right)$. If $X^{\prime} \rightarrow X$ is an fppf cover, $H^{1}\left(X^{\prime} / X, G\right)$ can be computed by means of cocycles (just as in Galois cohomology), so that

\footnotetext{
${ }^{3} \mathrm{An}$ fppf cover is thus a covering morphism (morphism couvrant) for the fppf topology in the sense of [SGA3], but not conversely.
} 
$H^{1}\left(X^{\prime} / X, G\right)$ can be identified with a subset of $\check{H}^{1}\left(X^{\prime} / X, G\right)$. If $G$ is affine and locally of finite presentation over $X$ then $H^{1}\left(X^{\prime} / X, G\right)=\check{H}^{1}\left(X^{\prime} / X, G\right)$, and the natural map

$$
H^{1}(X, G) \rightarrow \check{H}^{1}(X, G)
$$

is bijective. This will be the situation that we will consider in our paper, and we will indistinctively think of (the isomorphism class) of a torsor as an element of $H^{1}$ or the corresponding $\check{H}^{1}$. Along similar lines we write $Z^{1}$ instead of $\check{Z}^{1}$.

3.2. Remark. Assume that $G$ is an algebraic group over $k$. Following standard practice, we will denote in what follows $H^{1}\left(X, G_{X}\right)$ simply by $H^{1}(X, G)$. Because $G$ is of finite type, any $X$-torsor under $G$ that becomes trivial over $X_{s}$ already becomes trivial over $X_{k^{\prime}}$ for some finite Galois extension $k^{\prime} \subset k_{s}$ of $k$ (the extension $k^{\prime}$ depends of course on the given torsor). As a consequence, the natural map

$$
\underset{k^{\prime} / k \text { Galois }}{\lim _{\longrightarrow}} H^{1}\left(X_{k^{\prime}} / X, G\right) \rightarrow H^{1}\left(X_{s} / X, G\right)
$$

is bijective. Since $X_{k^{\prime}}$ is a Galois extension of $X$ whose Galois group is naturally isomorphic to $\operatorname{Gal}\left(k^{\prime} / k\right)$, we have $H^{1}\left(X_{k^{\prime}} / X, G\right) \simeq H^{1}\left(\operatorname{Gal}\left(k^{\prime} / k\right), G\left(X_{k^{\prime}}\right)\right)$. We thus have a bijection

$$
\left.H^{1}\left(X_{s} / X, G\right) \simeq H^{1}\left(\operatorname{Gal}\left(k_{s} / k\right), G\left(X_{s}\right)\right)\right),
$$

where the $H^{1}$ on the right denotes the "usual" Galois cohomology of the profinite group $\operatorname{Gal}\left(k_{s} / k\right)$ acting (continuously) on the (discrete) module $G\left(X_{s}\right)$. The natural map $\eta: H^{1}(k, G) \longrightarrow H^{1}(X, G)$ corresponds to the composition of maps in the sequence

$H^{1}\left(\operatorname{Gal}\left(k_{s} / k\right), G\left(k_{s}\right)\right) \rightarrow H^{1}\left(\operatorname{Gal}\left(k_{s} / k\right), G\left(X_{s}\right)\right) \simeq H^{1}\left(X_{s} / X, G\right) \subset H^{1}(X, G)$, where the first map arises from the inclusion $G\left(k_{s}\right) \subset G\left(X_{s}\right)$ obtained from the $k_{s}$-scheme structure of $X_{s}$.

3.3. Twisting. Throughout this section $X$ will denote a $k$-scheme, and $G$ a group scheme over $X$ that we assume is affine and locally of finite presentation over $X$. Let $\operatorname{Aut}(G)$ be the $X$-group functor of automorphisms of $G$ : For each $Y / X$

$$
\operatorname{Aut}(\mathbf{G})(Y)=\operatorname{Aut}\left(G_{Y}\right)
$$

The functor $\operatorname{Aut}(G)$ is always a sheaf of groups for the fppf topology on $X$, but it need not in general be representable (i.e. a group scheme).

Let $Y \rightarrow X$ be an fppf cover. To a cocycle $z \in \check{Z}^{1}(Y / X$, Aut $(G)) \subset$ $\left.\operatorname{Aut}\left(G_{Y \times X} Y\right)\right)$ one can associate a twisted group scheme ${ }_{z} G$ over $X$ whose functor of points is given by

$$
{ }_{z} G(S)=\left\{x \in G\left(Y \times_{X} S\right) \mid z^{S}\left(p_{1}^{S}(x)\right)=p_{2}^{S}(x)\right\}
$$

for any $X$-scheme $S$. The notation in (3.4) is as follows. The morphisms $p_{i}^{S}: Y \times_{X} Y \times_{X} S \rightarrow Y \times_{X} S$ are given by $p_{i}^{S}=p_{i} \times \mathrm{id}_{S}$. Given $x \in$ 
$G\left(Y \times_{X} S\right)=\operatorname{Hom}_{X}\left(Y \times_{X} S, G\right)$ we denote by $p_{i}^{S}(x)$ the composition $x \circ p_{i}^{S}$. The $p_{i}^{S}(x)$ are thus elements of the abstract group $G\left(Y \times_{X} Y \times_{X} S\right)$. Finally, since $z$ is an automorphism of the $Y \times_{X} Y$-group $G_{Y \times_{X} Y}$, it induces an automorphism $z^{S}$ of the abstract group $G\left(Y \times_{X} Y \times_{X} S\right)$.

We can reinterpret this definition by saying that the sequence

$$
{ }_{z} G(S) \subset G\left(Y \times_{X} S\right) \underset{p_{2}^{S}}{\stackrel{z^{S} \circ p_{1}^{S}}{\longrightarrow}} G\left(Y \times_{X} Y \times_{X} S\right)
$$

is exact. If $z=1$ then the twisted group ${ }_{z} G$ is isomorphic to $G$, and the sequence

$$
G(S) \subset G\left(Y \times_{X} S\right) \underset{p_{2}^{S}}{\stackrel{p_{1}^{S}}{\longrightarrow}} G\left(Y \times_{X} Y \times_{X} S\right)
$$

is exact. This allows us to identify $G(S)$ with a subgroup $G\left(Y \times_{X} S\right)$; an identification that we will henceforth use, whenever convenient, without further reference.

Since $z \in \check{Z}^{1}(Y / X$, Aut $(G))$ the group schemes ${ }_{z} G_{Y}$ and $G_{Y}$ are isomorphic. This isomorphism can be made explicit at the level of functor of points as we now explain for future reference.

If $i, j, k \in\{1,2,3\}$ are different integers and $j<k$ we set

$$
z_{i}=p_{j k}(z) \in \operatorname{Aut}\left(G_{Y \times_{X} Y \times_{X} Y}\right) .
$$

Given any scheme $S$ over $Y$ our automorphism $z$ induces naturally an automorphism $z^{S / Y}$ of the (abstract) group $G\left(Y \times_{X} Y \times_{Y} S\right)$. Similarly the $z_{i}$ induce automorphisms $z_{i}^{S / Y}$ of the (abstract) group $G\left(Y \times_{X} Y \times_{X} Y \times_{Y} S\right)$. By taking the composite map $S \rightarrow Y \rightarrow X$ we may also view $S$ as a scheme over $X$. The canonical isomorphism $Y \times_{Y} S \simeq S$ yields canonical identifications

$$
\begin{array}{r}
G(S) \simeq G\left(Y \times_{Y} S\right) \\
G\left(Y \times_{X} S\right) \simeq G\left(Y \times_{X} Y \times_{Y} S\right), \\
G\left(Y \times_{X} Y \times_{X} S\right) \simeq G\left(Y \times_{X} Y \times_{X} Y \times_{Y} S\right) .
\end{array}
$$

For $i=1,2$ we denote $p_{i 3} \times \operatorname{id}_{S}: Y \times_{X} Y \times_{X} Y \times_{Y} S \rightarrow Y \times_{X} Y \times_{Y} S$ by $p_{i 3}^{S / Y}$. Consider the diagram 


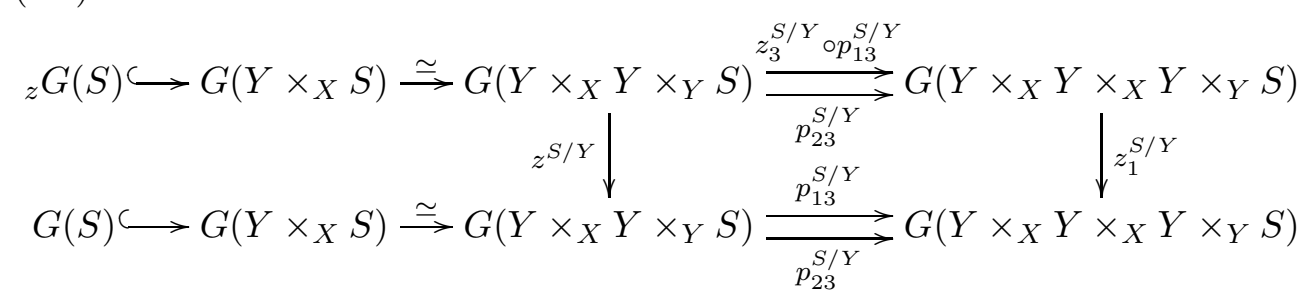

The top row is the equalizer (3.5) combined with the identifications of (3.7), and the bottom row arises in the same fashion for the trivial cocycle as described in (3.6). Since $z$ is a cocycle we have $z_{2}=z_{1} z_{3}$. This yields $z_{2}^{S / Y}=z_{1}^{S / Y} z_{3}^{S / Y}$ which shows that the outermost right square is commutative. That the innermost right square is commutative is easy. This shows that $z^{S / Y}$ induces a group isomorphism $z G(S) \rightarrow G(S)$. This isomorphism is functorial on $Y$-schemes $S$ and thus defines a $Y$-group scheme isomorphism which we still denote by $z$ :

$$
z:{ }_{z} G_{Y} \rightarrow G_{Y}
$$

Finally, if $\lambda:{ }_{z} G(X) \rightarrow{ }_{z} G(Y)$ denotes the canonical inclusion corresponding to our fppf-cover $Y / X$, one can easily checks that the diagram

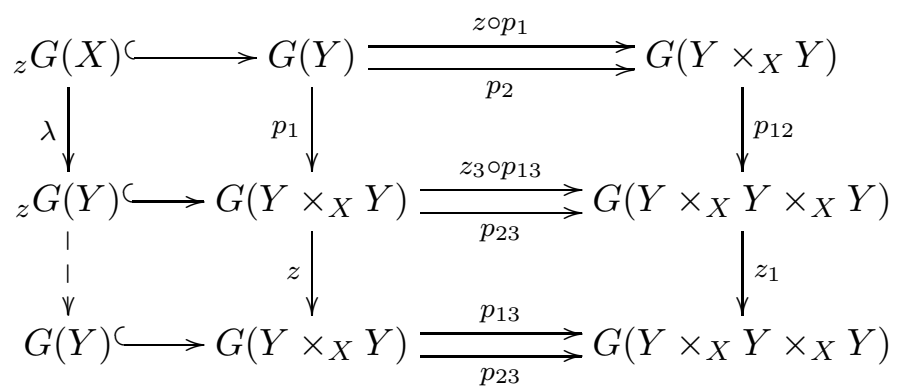

commutes. Here the first row is the equalizer (3.6) for $S=X$, and the last two rows constitute (3.8) in the case $Y=S$.

3.10. Remark. Let $Y \rightarrow X$ be an fppf cover, and let $z \in \check{Z}^{1}(Y / X, G) \subset$ $G\left(Y \times_{X} Y\right)$ be a cocycle. The element $z$ defines an (inner) automorphism $\operatorname{int}(z)$ of the group $G_{Y \times_{X} Y}$. It is clear that $\operatorname{int}(z) \in \check{Z}^{1}(Y / X$, Aut $(G))$. We denote the corresponding twisted $\operatorname{group} \operatorname{int}(z) G$ by ${ }_{z} G$.

We now turn our attention to maximal tori. For convenience we will denote the twisted group ${ }_{z} G$ by $G^{\prime}$, and let $\mathcal{T}$ and $\mathcal{T}^{\prime}$ denote the scheme of maximal tori of $G$ and $G^{\prime}$ respectively [SGA3, XI.4]. We recall that $\mathcal{T}$ and $\mathcal{T}^{\prime}$ are affine schemes of finite type over $X$ (ibid.). The cocycle $z$ acts naturally on the scheme $\mathcal{T}_{Y \times Y}$ of maximal tori of $G_{Y \times Y}$. We denote (by a slight abuse of notation) the resulting automorphism of $\mathcal{T}_{Y \times Y}$ also by $z$. In this way we may view $z$ as a cocycle in $\check{Z}^{1}(Y / X, \operatorname{Aut}(\mathcal{T}))$. By [SGA3]Exp. 
XXIV prop.4.2.1 $\mathcal{T}^{\prime}$ coincides with the twist of $\mathcal{T}$ by $z$, i.e., we may assume that the functor of points of $\mathcal{T}^{\prime}$ is given by

$$
\mathcal{T}^{\prime}(S)=\left\{T^{\prime \prime} \in \mathcal{T}(Y \times S) \mid z\left(p_{1}^{S}\left(T^{\prime \prime}\right)\right)=p_{2}^{S}\left(T^{\prime \prime}\right)\right\} .
$$

Arguing as above we get the commutative diagram

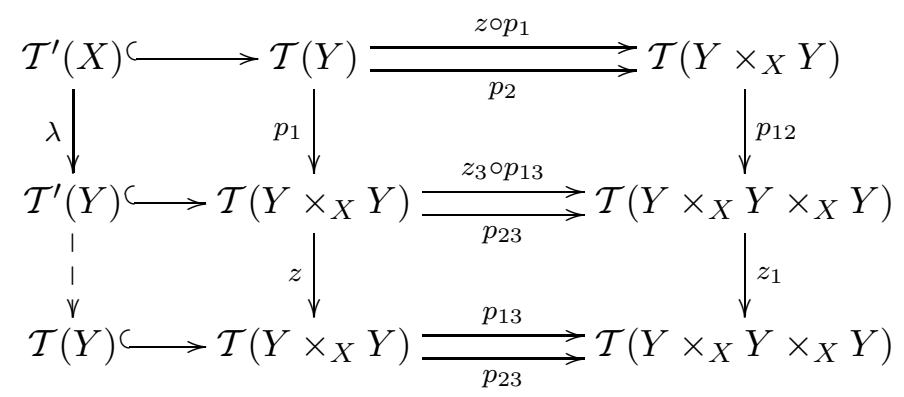

Just as in (3.9), the automorphism $z$ induces a group isomorphism (also denoted by $z)$

$$
z: \mathcal{T}_{Y}^{\prime} \rightarrow \mathcal{T}_{Y}
$$

The following result is inevitable.

3.14. Lemma. Let $T^{\prime}$ be a maximal $X$-torus of $G^{\prime}$ (i.e., $T^{\prime} \in \mathcal{T}^{\prime}(X)$ ), and let $T^{\prime \prime}$ be the corresponding maximal torus of $G_{Y}$ given by (3.11). Then $z\left(T_{Y}^{\prime}\right)=T^{\prime \prime}$.

Proof. We have $\lambda\left(T^{\prime}\right)=T_{Y}^{\prime}$, so we need to show that $z\left(\lambda\left(T^{\prime}\right)\right)=T^{\prime \prime}$. Let $T^{\prime \prime \prime} \in \mathcal{T}(Y)$ be the image of $\lambda\left(T^{\prime}\right)$ under $z$ given by (3.12). The inclusion $\mathcal{T}(Y) \subset \mathcal{T}\left(Y \times_{X} Y\right)$ in the bottom row of (3.12) is given by the projection $p_{2}$. Thus $z\left(p_{1}\left(T^{\prime \prime}\right)\right)=p_{2}\left(T^{\prime \prime \prime}\right)$. By the top row of $(3.12)$ we have $z\left(p_{1}\left(T^{\prime \prime}\right)\right)=$ $p_{2}\left(T^{\prime \prime}\right)$. It follows that $T^{\prime \prime}=T^{\prime \prime \prime}$ as desired

3.15. Lemma. Let $G$ be a reductive group scheme over $X$, and $T$ a maximal torus of $G$ (assumed to exist). Let $Y / X$ be an fppf cover of $X, z \in$ $\check{Z}^{1}(Y / X, G)$ a cocycle. If the twisted $X$-group ${ }_{z} G$ admits a maximal torus $T^{\prime}$ such that $T_{Y}^{\prime}$ and $T_{Y}$ are conjugate by an element of $G(Y)$ (where ${ }_{z} G_{Y}$ is identified with $G_{Y}$ as described in (3.9) above), then

$$
[z] \in \operatorname{Im}\left[H^{1}\left(Y / X, N_{G}(T)\right) \longrightarrow H^{1}(X, G)\right] .
$$

Proof. To begin with we recall that $N_{G}(T)$ is a closed smooth subgroup of $G$ ([SGA3] Exp XI.5 and XIX.1) As before we denote the twisted group ${ }_{z} G$ by $G^{\prime}$, and let $\mathcal{T}$ and $\mathcal{T}^{\prime}$ denote the scheme of maximal tori of $G$ and $G^{\prime}$ respectively.

According to 3.11 our torus $T^{\prime}$ of $G^{\prime}$ corresponds to a torus $T^{\prime \prime}$ of $G_{Y}$. By Lemma $3.14 T_{Y}^{\prime}$ coincides with $T^{\prime \prime}$ via our identification $G_{Y}^{\prime} \simeq G_{Y}$. Hence $T^{\prime \prime}=g^{-1} T g$ for some $g \in G(Y)$. Let $z^{\prime}=p_{2}(g) z p_{1}(g)^{-1}$. Then $z^{\prime} \in G\left(Y \times_{X} Y\right)$ is a cocycle equivalent to $z$. We have

$$
z p_{1}\left(T^{\prime \prime}\right) z^{-1}=p_{2}\left(T^{\prime \prime}\right)
$$


because $T^{\prime} \in \mathcal{T}^{\prime}(X)$. This implies that

$$
\begin{gathered}
z p_{1}\left(g^{-1} T_{Y} g\right) z^{-1}=p_{2}\left(g^{-1} T_{Y} g\right) \text { or } \\
z p_{1}(g)^{-1} p_{1}\left(T_{Y}\right) p_{1}(g) z^{-1}=p_{2}(g)^{-1} p_{2}\left(T_{Y}\right) p_{2}(g), \text { or } \\
z^{\prime} p_{1}\left(T_{Y}\right)\left(z^{\prime}\right)^{-1}=p_{2}\left(T_{Y}\right) .
\end{gathered}
$$

Because $T$ is defined over $X$ this last yields

$$
z^{\prime} T_{Y{ }_{X} Y}\left(z^{\prime}\right)^{-1}=T_{Y{ }_{X} Y}
$$

Thus $z^{\prime} \in N_{G}(T)\left(Y \times_{X} Y\right)$.

3.4. Reducibility and isotropy. Let $G$ be a reductive group scheme over a base scheme $X$. We recall two fundamental notions about $\mathrm{G}$; one global (reducibility), and the other local (isotropy).

We say that $G$ is reducible if $G$ admits a proper parabolic subgroup, and irreducible otherwise. We denote by $\operatorname{Par}(G)$ the $X$-scheme of parabolic subgroup schemes of $G$ [SGA3, XXVI.3.5]. This scheme is smooth and projective over $X$. Since by definition $G$ is a parabolic subgroup of $G$, to say that $G$ is reducible is to say that $\operatorname{Par}(G)(X) \neq\{G\}$.

3.16. Remark. If $X$ is connected, to each parabolic subgroup $P$ of $G$ corresponds a "type" $\mathbf{t}=\mathbf{t}(P)$ which is a subset of the Dynkin diagram of $G$. Given a type $\mathbf{t}$ the scheme $\operatorname{Par}_{\mathbf{t}}(G)$ of parabolic subgroups of $G$ of type $\mathbf{t}$ is also smooth and proper over $X$.

We know that if $G$ contains a non-central split subtorus, then $G$ is reducible (loc. cit., 6.3). As we will presently see, the converse is true locally.

Assume that the base scheme $X$ is semilocal and connected. Following [SGA3, XXVI.6.13] we say that $G$ is isotropic if (as a reductive $X$-group) $G$ admits a non-trivial split subtorus. Otherwise we say that $G$ is anisotropic. Recall that the radical torus $\operatorname{rad}(G)$ is the unique maximal torus of the center of $G$ [SGA3, XXII.4.3.6].

3.17. Proposition. Assume that $X$ is semilocal connected.

(1) [SGA3, XXVI.6.12] The following are equivalent:

(a) $G$ is reducible.

(b) $G$ admits a non-central split subtorus.

(2) [SGA3, XXVI.6.14] The following are equivalent:

(a) $G$ is isotropic.

(b) $G$ is reducible or $\operatorname{rad}(G)$ is isotropic. 
3.5. Patching group schemes. A technical tool used by Raghunathan and Ramanathan in their proof falls within the content of the following useful result.

3.18. Lemma. Let $X$ be an algebraic curve over $k$ (i.e. a one-dimensional separated irreducible algebraic scheme over $k$ ), and let $K$ denote its function field (i.e. the local ring of its generic point). Let $x \in X$ be a closed point, and assume that the local ring $D=O_{X, x}$ is a discrete valuation ring (for example, if $X$ is smooth). Let $\widehat{D}$ and $\widehat{K}$ denote the corresponding completions.

Assume we are given a triple $(G, F, \tau)$ consisting of:

(i) An affine group scheme $G$ over $U=X-\{x\}$ of finite type.

(ii) An affine and finitely presented group scheme $F$ over $\widehat{D}$.

(iii) A $\widehat{K}$-group scheme isomorphism $\tau: G \times_{U} \widehat{K} \simeq F \times_{\widehat{D}} \widehat{K}$.

Then there exists a group scheme $H$, affine and of finite type over $X$, such that $H \times_{X} U=G$ and $H \times_{X} \widehat{D}=F$. Furthermore, if $G$ and $F$ are smooth, then so is $H$.

Proof. By [BLR] $\S 6.2$ proposition D.4(b) applied to our isomorphism $\tau$ : $\left(G \times_{U} K\right) \times_{K} \widehat{K} \simeq F \times_{\widehat{D}} \widehat{K}$ there exists a group scheme $F_{D}$ over $D$ together with isomorphisms

(a) $F_{D} \times_{D} \widehat{D} \simeq F$ and

(b) $F_{D} \times{ }_{D} K \simeq G \times \times_{U} K$.

which are compatible with $\tau$. Note that since $D \rightarrow \widehat{D}$ is faithfully flat the descended group $F_{D}$ is finitely presented.

Fix an affine open neighborhood $\operatorname{Spec}(S)$ of $x$. Since $D=S_{x}$ and $F_{D}$ is finitely presented, there exists $f \in S$ with $f(x) \neq 0$ [i.e. $x \in \operatorname{Spec}\left(S_{f}\right)$ ] and a finitely presented $S_{f}$-group $F_{f}$ such that $F_{f} \times S_{f} D \simeq F_{D}$.

Choose $g \in S$ such that $\operatorname{Spec}\left(S_{f g}\right) \subset \operatorname{Spec}(S) \cap U$. If we set $G_{f g}=G \times_{U} S_{f g}$ and $F_{f g}=F_{f} \times_{S_{f}} S_{f g}$ then (b) above yields an isomorphism of $K$-groups

$$
G_{f g} \times_{S_{f g}} K \simeq F_{f g} \times_{S_{f g}} K .
$$

Because both $G_{f g}$ and $F_{f g}$ are of finite type and $K$ is the field of quotients of $S_{f g}$, there exists some $h \in S$ such that

$$
G \times_{U} S_{f g h} \simeq F_{f} \times_{S_{f}} S_{f g h} .
$$

Let $V=\operatorname{Spec}\left(S_{f g h}\right) \cup\{x\} \subset \operatorname{Spec}\left(S_{f}\right)$. Then the $U$-group $G$ and the $V$ group $F_{f} \times_{S_{f}} V$ are isomorphic on the overlap $U \cap V=\operatorname{Spec}\left(S_{f g h}\right)$. We can thus glue these two groups to obtain a group $H$ over $X$ such that $H_{U} \simeq G$ and $H_{\widehat{D}} \simeq F$.

Let $Y=U \bigsqcup \operatorname{Spec} \widehat{D}$. The natural map $Y \rightarrow X$ is faithfully flat and quasicompact. The assertions about $H$ being affine and of finite type, and smooth if $G$ and $F$ are smooth, now follow from descent ([EGA4] II Prop. 2.7.1 and IV Cor. 17.7.3(ii)). 
The group $H$ constructed above is said to correspond to the given triple $(G, F, \tau)$. The following two lemmas easily follow from the above argument and properties of descent in the faithfully flat quasicompact topology.

3.19. Lemma. Assume we are given two triples $(G, F, \tau)$ and $\left(G^{\prime}, F^{\prime}, \tau^{\prime}\right)$ as in the previous Lemma. If $\phi_{1}: G^{\prime} \rightarrow G$ and $\phi_{2}: F^{\prime} \rightarrow F$ are isomorphisms over $U$ and $\widehat{D}$ respectively such that the diagram

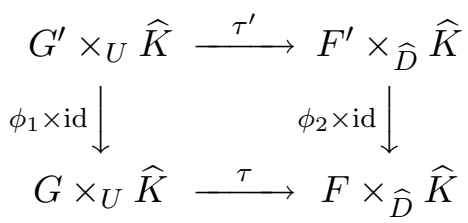

commutes, then the group schemes $H$ and $H^{\prime}$ over $X$ corresponding to $(G, F, \tau)$ and $\left(G^{\prime}, F^{\prime}, \tau^{\prime}\right)$ are isomorphic.

3.20. Lemma. Assume that we are given a triple $(G, F, \tau)$ as in Lemma 3.18. If $G^{\prime}<G$ and $F^{\prime}<F$ are closed subgroup schemes such that $\tau\left(G^{\prime} \times_{U} \widehat{K}\right)=F^{\prime} \times_{\widehat{D}} \widehat{K}$, then the group scheme $H^{\prime}$ corresponding to the triple $\left(G^{\prime}, F^{\prime}, \tau_{\left.\right|_{G^{\prime} \times} \widehat{K}}\right)$ admits a natural closed immersion into the group scheme $H$ corresponding to $(G, F, \tau)$.

3.21. Example. The following example, which gives a procedure for extending certain group schemes over $\mathbb{A}_{k}^{1}$ to the projective line $\mathbb{P}_{k}^{1}$, is of fundamental importance to the proof of the main theorems.

We maintain the general notation of the previous Lemmata, but look at the particular case when $X$ is the projective line $\mathbb{P}_{k}^{1}$ over $k, U=\mathbb{A}_{k}^{1}=$ $\operatorname{Spec}(k[t])$ is the affine line over $k$, and $\{x\}=X \backslash U$ is the point at infinity of $X$. We have $D=\mathcal{O}_{P_{k}^{1}, x}=\operatorname{Spec}\left(k\left[\frac{1}{t}\right]_{\left(\frac{1}{t}\right)}\right), K=k(t)=k\left(\frac{1}{t}\right)$ where $t$ is our coordinate function on $U, \widehat{D}=k\left[\left[\frac{1}{t}\right]\right]$ and $\widehat{K}=k\left(\left(\frac{1}{t}\right)\right)$.

(a) Let $G=\operatorname{Spec}(k[t][G])$ be a semisimple simply connected group scheme over $U$. Consider the generic fiber $G_{K}$ of $G$ and pass to the completion $G_{\widehat{K}}$. Let $p$ be a point of the building $\mathcal{B}=\mathcal{B}_{G_{\widehat{K}}}$ corresponding to $G_{\widehat{K}}$. Recall that $G(\widehat{K})$ acts on $\mathcal{B}$, and that the stabilizer $\operatorname{Stab}_{G(\widehat{K})}(p)$ of $p$ in $G(\widehat{K})$ is the parahoric subgroup of $G(\widehat{K})$ associated to $p$. This parahoric subgroup, in turn, gives rise to a smooth group scheme $F$ over $\widehat{D}$ whose generic fiber is canonically isomorphic to $G_{\widehat{K}}[\mathrm{BT} 2, \S 5.1 .8]$ :

$$
\tau: G \times_{k[t]} \widehat{K} \rightarrow F \times_{\widehat{D}} \widehat{K} .
$$

More precisely, Bruhat-Tits theory (ibid.) shows that $F=\operatorname{Spec}(\widehat{D}[F])$ where

$$
\widehat{D}[F]=\left\{f \in \widehat{K}[G] \mid f(x) \in \widehat{D} \text { for all } x \in \operatorname{Stab}_{G(\widehat{K})}(p)\right\}
$$


Moreover $\widehat{D}[F] \otimes_{\widehat{D}} \widehat{K}=\widehat{K}[G]$, and this gives rise to the isomorphism $\tau$ of (3.22). The triple $(G, F, \tau)$ corresponds then to the diagram

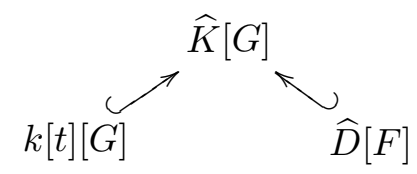

Of course, by Lemma 3.18, this data gives rise to a group scheme $H=H(p)$ which is affine and smooth over $\mathbb{P}_{k}^{1}$.

(b) For future use we note that the above construction of $F$ (and hence of $H)$ is compatible with any finite Galois field extension $k^{\prime} / k$ [BT2, §5]. More precisely, if $K^{\prime}=k^{\prime}\left(\frac{1}{t}\right)$ then the extension $\widehat{K}^{\prime} / \widehat{K}$ is unramified, so it gives rise to a canonical embedding $\mathcal{B}_{G_{\widehat{K}}} \hookrightarrow \mathcal{B}_{G_{\widehat{K}^{\prime}}}$ of the buildings corresponding to $G_{\widehat{K}}$ and $G_{\widehat{K}}$, respectively. Let $F^{\prime}$ denote the group scheme over $\widehat{D}^{\prime}=k^{\prime}\left[\left[\frac{1}{t}\right]\right]$ associated to $p$ viewed now as an element $p^{\prime}$ of $\mathcal{B}_{G_{\widehat{K}^{\prime}}}$. Then we have $F \times \widehat{D}$ $\widehat{D}^{\prime} \simeq F^{\prime}$ via $\widehat{D^{\prime}}\left[F^{\prime}\right]=\widehat{D}[F] \otimes_{\widehat{D}} \widehat{D^{\prime}}$. It follows that $H\left(p^{\prime}\right) \simeq H(p) \times_{\mathbb{P}_{k}^{1}} \mathbb{P}_{k^{\prime}}^{1} \simeq$ $H(p) \times_{k} k^{\prime}$.

(c) Assume additionally that $G$ is of the form $G=G_{0} \times_{k} \mathbb{A}_{k}^{1}$ where $G_{0}$ is a $k$-split group and that $p$ is contained in the apartment $\mathcal{A} \subset \mathcal{B}$ corresponding to a maximal $k$-split torus $T_{0} \subset G_{0}$. Then $T_{0}(k)$ acts trivially on $p$ and this gives rise to the canonical closed embedding

$$
T_{0, \widehat{D}}=T_{0} \times_{k} \widehat{D} \hookrightarrow F
$$

of $\widehat{D}$-groups. Since $T_{0} \subset G_{0}$ we also have the canonical closed embedding

$$
T_{0, k[t]}=T_{0} \times_{k} k[t] \hookrightarrow G=G_{0} \times_{k} k[t] .
$$

Both of the above embeddings are compatible with the isomorphism $\tau$ of (3.22), i.e.

$$
\tau\left(T_{0, k[t]} \times_{k[t]} \widehat{K}\right)=T_{0, \widehat{D}} \times_{\widehat{D}} \widehat{K} \subset F \times_{\widehat{D}} \widehat{K} .
$$

If we denote by $\tau^{\prime}$ the restriction of $\tau$ to $T_{0, k[t]} \times_{k[t]} \widehat{K} \leq G \times_{k[t]} \widehat{K}$, then by Lemma 3.20 , the triple $\left(T_{0, k[t]}, T_{0, \widehat{D}}, \tau^{\prime}\right)$ corresponds to a group subscheme $T \subset H$ over $\mathbb{P}_{k}^{1}$. Moreover, from the diagram

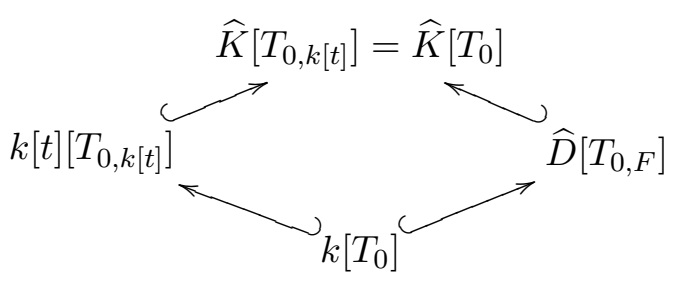

it follows that $T \simeq T_{0} \times_{k} \mathbb{P}_{k}^{1}$. In particular, $T$ is split.

(d) Let $g \in G(k[t]) \subset G(\widehat{K})$. Then $g$ yields an (inner) automorphism $\operatorname{int}(g) \in \operatorname{Aut}\left(G_{\widehat{K}}\right)$. Let $g^{\star}: \widehat{K}[G] \rightarrow \widehat{K}[G]$ be the comorphism corresponding 
to $\operatorname{int}(g)$. Set $p^{\prime}=g(p)$ and let $F^{\prime}$ be the group scheme over $\widehat{D}$ corresponding to $p^{\prime}$. Then we clearly have

$$
g^{\star}: \widehat{D}[F] \rightarrow \widehat{D}\left[F^{\prime}\right]=g^{\star}(\widehat{D}[F]) .
$$

Thus $g^{\star}$ gives rise to an isomorphism $\phi: F^{\prime} \rightarrow F$. Set

$$
\phi_{1}=\operatorname{int}(g): G=G^{\prime} \rightarrow G,
$$

and $\tau^{\prime}=\left(\phi \times \operatorname{id}_{\widehat{K}}\right)^{-1} \circ \tau \circ\left(\operatorname{int}(g) \times \operatorname{id}_{\widehat{K}}\right)$. By Lemma 3.18, the triple $\left(G, F^{\prime}, \tau^{\prime}\right)$ gives rise to a group scheme $H^{\prime}$ over $\mathbb{P}_{k}^{1}$, and by Lemma 3.19 we have $H^{\prime} \simeq H$. Tracing through all these constructions and identifications we see that $\tau^{\prime}$ corresponds to the diagram

$$
\overbrace{k[t][G]}^{\widehat{K}[G]} \overbrace{\widehat{D}\left[F^{\prime}\right]=g^{\star}(\widehat{D}[F])}
$$

3.6. Weil restriction considerations. Throughout this section $X$ will denote a projective variety over $k$, and $H$ a group scheme which is affine and of finite type over $X$.

For a given $k$-scheme $S$ we denote by $h_{S}$ the corresponding functor of points:

$$
\begin{gathered}
h_{S}: S c h / k \longrightarrow S e t s \\
Y \longrightarrow \operatorname{Hom}_{k}(Y, S) .
\end{gathered}
$$

Recall Grothendieck's definition of the Weil restriction of the $X$-scheme $H$ to $k .{ }^{4}$ [Gr, exp. 221, Remarque 3.9.c]. This is the functor

$$
\prod_{X / k} H: S c h / k \longrightarrow S e t s
$$

defined by

$$
Y \longrightarrow \operatorname{Hom}_{X}\left(X \times_{k} Y, H\right)
$$

According to loc. cit. (see also [H2, page 121]), this functor is representable by an affine $k$-scheme of finite type, say $S / k$, which we henceforth assume is fixed in our discussion. Fix an isomorphism

$$
\alpha: h_{S} \rightarrow \prod_{X / k} H
$$

By definition our map $\alpha$ is thus a family of bijections

$$
\operatorname{Hom}_{k}(Y, S) \stackrel{\alpha_{Y}}{\longrightarrow} \operatorname{Hom}_{X}\left(Y \times_{k} X, H\right)
$$

which is functorial on $k$-schemes $Y$. The identity $\operatorname{map} \operatorname{id}_{S}: S \rightarrow S$ defines a morphism

$$
\mathrm{ev}_{\alpha}: S \times_{k} X \longrightarrow H
$$

\footnotetext{
${ }^{4}$ Called the functor of global sections in $[R R]$.
} 
called the evaluation map. By Yoneda considerations the bijections of (3.27) are then given by

$$
\alpha_{Y}: f \rightarrow \mathrm{ev}_{\alpha} \circ\left(f \times \mathrm{id}_{X}\right) .
$$

The algebraic $k$-scheme $S$ can be made into a $k$-group by transport of structure via $\alpha$. More precisely, the group structure on $S(Y)$ is as follows: Given $a, b \in S(Y)$ then $a b=c$ where $c$ satisfies

$$
\alpha_{Y}(c)=\alpha_{Y}(a) \alpha_{Y}(b)
$$

where the right-hand side is the multiplication on the group $H\left(Y \times_{k} X\right)$. We denote this group by $S^{\alpha}$, or simply by $S$ if no confusion is possible. One checks that $\mathrm{ev}_{\alpha}$, when viewed as a map from $S^{\alpha} \times_{k} X$ to $H$ is in fact a morphism of group schemes over $X$.

3.30. Remark. Assume that the $X$-group $H$ is obtained by base change from an affine $k$-group $G_{0}$, i.e., that $H=G_{0} \times{ }_{k} X$. Then we may take $S$ to be $G_{0}$. Indeed, since $X / k$ is projective the canonical map $\mathcal{O}(Y) \rightarrow \mathcal{O}\left(X \times_{k} Y\right)$ is an isomorphism. Thus

$$
\begin{gathered}
\operatorname{Hom}_{k}\left(Y, G_{0}\right) \simeq \operatorname{Hom}_{k}\left(k\left[G_{0}\right], \mathcal{O}(Y)\right) \simeq \operatorname{Hom}_{k}\left(k\left[G_{0}\right], \mathcal{O}\left(Y \times_{k} X\right)\right) \simeq \\
\operatorname{Hom}_{k}\left(Y \times_{k} X, G_{0}\right) \simeq \operatorname{Hom}_{X}\left(Y \times_{k} X, G_{0} \times_{k} X\right) .
\end{gathered}
$$

Note that the resulting isomorphism $\alpha: h_{G_{0}} \rightarrow \prod_{X / k}\left(G_{0} \times_{k} X\right)$ is such that $\mathrm{ev}_{\alpha}=\mathrm{id}_{G_{0} \times_{k} X}$.

3.31. Remark. Let $k^{\prime}$ be a field extension of $k$ and set $X^{\prime}=X \times_{k} k^{\prime}$ and $H^{\prime}=H \times_{k} k^{\prime}$. Then the Weyl restriction $\prod_{X^{\prime} / k^{\prime}} H^{\prime}$ is represented by $S^{\prime}=S \times_{k} k^{\prime}$. A natural isomorphism

$$
\alpha^{\prime}: h_{S^{\prime}} \longrightarrow \prod_{X^{\prime} / k^{\prime}} H^{\prime}
$$

is obtained from $\alpha$ via the following functorial identifications on schemes $Y$ over $k^{\prime}$ :

$$
\begin{aligned}
& h_{S^{\prime}}(Y)=\operatorname{Hom}_{k^{\prime}}\left(Y, S^{\prime}\right) \simeq \operatorname{Hom}_{k}(Y, S)=h_{S}(Y) \\
& \stackrel{\alpha_{Y}}{\simeq} \operatorname{Hom}_{X}\left(Y \times_{k} X, H\right) \\
& \simeq \operatorname{Hom}_{k^{\prime} \times_{k} X}\left(Y \times_{k} X, k^{\prime} \times_{k} H\right) \\
& \simeq \operatorname{Hom}_{k^{\prime} \times_{k} X}\left(Y \times_{k^{\prime}}\left(k^{\prime} \times_{k} X\right), k^{\prime} \times_{k} H\right) \\
& =\operatorname{Hom}_{X^{\prime}}\left(Y \times_{k^{\prime}} X^{\prime}, H^{\prime}\right) .
\end{aligned}
$$

It follows from this explicit description that the evaluation map commutes with base field extension, namely that

$$
\mathrm{ev}_{\alpha^{\prime}}=\mathrm{ev}_{\alpha} \times \mathrm{id}_{k^{\prime}}
$$


or, more precisely, that the diagram

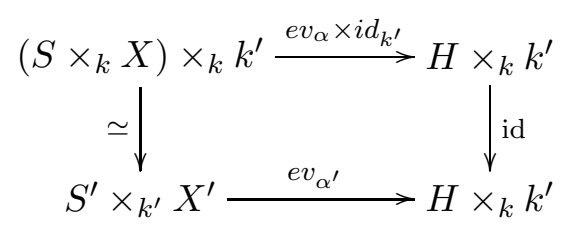

commutes.

3.32. Remark. If $\phi: H_{1} \rightarrow H_{2}$ is a morphism of $X$-groups we have a natural $k$-group morphism

$$
\prod \phi: \prod_{X / k} H_{1} \longrightarrow \prod_{X / k} H_{2}
$$

If $\phi$ is a monomorphism (i.e., if $\phi$ has trivial kernel) then $\prod \phi$ is also a monomorphism.

Assume our $\prod_{X / k} H_{i}$ are representable by algebraic $k$-schemes $S_{i}$ as explained above, and that we are given natural isomorphisms $\alpha_{i}: h_{S_{i}} \simeq$ $\prod_{X / k} H_{i}$. These yields a morphism of $k$-groups $h_{\phi}: S_{1}^{\alpha_{1}} \rightarrow S_{2}^{\alpha_{2}}$ which, at the level of functor of points, is given by

$$
h_{\phi}=\alpha_{2}^{-1} \circ\left(\prod \phi\right) \circ \alpha_{1}: h_{S_{1}} \longrightarrow h_{S_{2}}
$$

Note that if $\phi$ is a monomorphism then so is $h_{\phi}$.

By Yoneda considerations $h_{\phi}$ corresponds to the $k$-algebra homomorphism $h_{\phi}\left(i d_{S_{1}}\right): S_{1} \rightarrow S_{2}$. Chasing through the definitions we see that the diagram

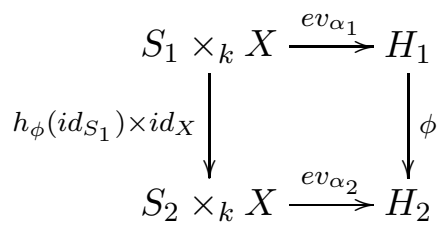

commutes. Of particular interest is the case when $H_{1}=T \times_{k} X$ for some $k$-group $T$. As we saw in Remark 3.30, we may take $S_{1}$ to be $T$ and the evaluation map $e v_{\alpha_{1}}: T \times_{k} X \rightarrow T \times_{k} X$ to be the identity. The above then reads

$$
\phi=e v_{\alpha_{2}} \circ\left(h_{\phi}\left(i d_{T}\right) \times i d_{X}\right) .
$$

3.33. Remark. The algebraic $k$-scheme $S$ need not be reduced. Following the Appendix we associate to $S$ a closed $k$-subscheme $S_{r}$ which is absolutely reduced. The $k$-group structure of $S^{\alpha}$ carries to $S_{r}$, and we denote the resulting algebraic $k$-group by $S_{r}^{\alpha}$, or simply by $S_{r}$ when no confusion is possible. If $Y$ is an absolutely reduced scheme over $k$ then every morphism $Y \rightarrow S$ factors through the closed immersion $S_{r} \hookrightarrow S$ (see the Appendix for details). We call the composition

$$
S_{r} \times_{k} X \hookrightarrow S \times_{k} X \stackrel{e v_{\alpha}}{\longrightarrow} H
$$

the (reduced) evaluation map and denote it by the symbol $e v_{\alpha}^{r}$. 
The main result of this section concern the rank of the algebraic $k$-group $S_{r}$.

3.34. Proposition. Let $H$ and $S$ be as above. Assume that $X \times_{k} k_{s}$ is connected. Let $M_{0}$ be a k-subgroup of multiplicative type of $S$.

(1) The morphism of $X$-group schemes $M_{0} \times_{k} X \rightarrow H$ induced by $e v_{\alpha}$ is a closed immersion.

(2) For all $x \in X$, we have $\operatorname{rank}\left(S_{r}\right) \leq \operatorname{rank}\left(H_{x}\right)$.

Proof. (1) Since the evaluation map commutes with base change (Remark 3.31 ) we may assume that $k=k_{s}$. Let $\mu$ denote the kernel of the $X$-group morphism $e v_{\alpha}: M_{0} \times_{k} X \rightarrow H$ induced by our evaluation map. According to theorem IX.6.8 of [SGA3] $\mu$ is a group scheme of multiplicative type and of finite type over $X$. Furthermore, to establish (1) it suffices to show that $\mu$ is trivial.

Since $k$ is separably closed $M_{0}$ is a diagonalisable $k$-group of finite type (prop. X.1.4 of [SGA3]). By Proposition IX.2.11.i of [SGA3], it follows that $\mu$ is diagonalisable as well (it is here that we use the assumption that $X_{s}$ is connected). Hence $\mu=\mu_{0} \times_{k} X$ where $\mu_{0}$ is a diagonalisable $k$-group of finite type. But by rigidity of diagonalisable groups ([SGA3], VIII.1.6), we have

$$
\operatorname{Hom}_{k-g r}\left(\mu_{0}, M_{0}\right) \stackrel{\sim}{\longrightarrow} \operatorname{Hom}_{X-g r}\left(\mu_{0} \times_{k} X, M_{0} \times_{k} X\right) .
$$

So we are given actually a morphism $\iota_{0}: \mu_{0} \rightarrow M_{0}$ which is a closed immersion.

Recall that $\prod_{X / k}\left(\mu_{0} \times_{k} X\right)$ is represented by $\mu_{0}$ with the identity map for evaluation map (see Remark 3.30). Similarly for $M_{0} \times_{k} X$ and $S \times_{k} X$. By Remark 3.32) if we take the Weil restriction for the $X$-group morphism $e v_{\alpha}: S \times_{k} X \rightarrow H$, then the corresponding $k$-group morphism $S \rightarrow S$ is the identity map. If we apply these considerations to the composite $X$-group morphism

$$
f: \mu_{0} \times_{k} X \stackrel{\iota=\iota_{0} \times \mathrm{id}}{\longrightarrow} M_{0} \times_{k} X \rightarrow S \times_{k} X \stackrel{e v_{\alpha}}{\longrightarrow} H
$$

we obtain

$$
f_{0}: \mu_{0} \stackrel{\iota_{0}}{\longrightarrow} M_{0} \hookrightarrow S \stackrel{\mathrm{id}}{\longrightarrow} S,
$$

which is a closed immersion. Since $\mu=\mu_{0} \times_{k} X$ the morphism $f$ of (3.35) is trivial. This forces the closed immersion $f_{0}$ of (3.36) to be trivial. Thus $\mu_{0}=1$, and consequently $\mu=1$ as desired.

(2) Let $T$ be a maximal $k$-torus of $S_{r}$. By (1), the $X$-group morphism $T \times{ }_{k} X \rightarrow H$ is a closed immersion. It follows that the morphism $T \times_{k} k(x) \rightarrow$ $H_{x}$ is a closed immersion for all points $x \in X$. Thus $\operatorname{rank}\left(S_{r}\right)=\operatorname{rank}(T) \leq$ $\operatorname{rank}\left(H_{x}\right)$. 


\section{TORSORS OVER THE AFFINE LINE}

Let $E$ be a geometrically separably trivial $\mathbb{A}_{k}^{1}$-torsor under the action of a $k$-linear algebraic group $G$ whose connected component of the identity is reductive. We must show that $E$ is constant.

We begin our proof by reducing to the case when $G$ is reductive.

4.1. Lemma. Let $G$ be a reductive $k$-group and $T$ a maximal torus of $G$. Suppose $X$ is a geometrically irreducible $k$-scheme for which the canonical map $k_{s}^{\times} \rightarrow \mathcal{O}_{X_{s}}\left(X_{s}\right)^{\times}$is an isomorphism. Then every geometrically separably trivial $X$-torsor under $N_{G}(T)$ is constant.

Proof. Let $N=N_{G}(T)$. The bijection (3.3) of Remark 3.2 shows that to a geometrically separably trivial $X$-torsor $E$ under $N$ corresponds the class of a (continuous) cocycle $u \in Z^{1}\left(\operatorname{Gal}\left(k_{s} / k\right), N\left(X_{s}\right)\right)$.

Since $T_{s}$ is split the underlying scheme structure of $N_{s}$ is given by $N_{s}=$ $\bigsqcup_{w \in W} \mathrm{G}_{m}^{l}$ where $\mathrm{G}_{m}$ denotes the multiplicative group over $k, l=\operatorname{rk}(G)$ and $W$ is the (abstract) Weyl group of $\left(G_{s}, T_{s}\right)$. Since $X$ is geometrically irreducible we obtain

$$
\begin{gathered}
N\left(X_{s}\right)=N_{s}\left(X_{s}\right)=\operatorname{Hom}\left(X_{s}, N_{s}\right)= \\
\bigsqcup_{w \in W} \mathrm{G}_{m}^{l}\left(X_{s}\right)=\bigsqcup_{w \in W} \mathrm{G}_{m}^{l}\left(k_{s}\right)=N\left(k_{s}\right) .
\end{gathered}
$$

Thus $u \in Z^{1}\left(\Gamma, N\left(k_{s}\right)\right)$ and $E$ is constant.

By considering the case when $X=\mathbb{A}_{k}^{1}$ and $G=T$ we obtain a stronger version of Theorem 2.1 for tori.

4.2. Corollary. Let $T$ be a k-torus. Every $\mathbb{A}_{k}^{1}$-torsor under $T$ is constant.

Proof. Since $T$ splits over $k_{s}$ and $\mathbb{A}_{k}^{1}$ has trivial Picard group, every $\mathbb{A}_{k}^{1}$-torsor under $T$ is geometrically separably trivial.

4.3. Proposition. Assume that every geometrically separably trivial $\mathbb{A}_{k}^{1}$ torsor under a reductive $k$-group is constant. If $G$ is an algebraic $k$-group whose connected component $G^{\circ}$ is reductive then every geometrically separably trivial $\mathbb{A}_{k}^{1}$-torsor under $G$ is also constant.

Proof. Let $E$ be a $G$-torsor over $\mathbb{A}_{k}^{1}$ which is trivial over $\mathbb{A}_{s}^{1}$. Consider the fiber $E_{0}$ of $E$ at the origin $0 \in \mathbb{A}_{k}^{1}$, and its class $\left[E_{0}\right] \in H^{1}(k, G)$. We view $E_{0}$ as a constant torsor over $\mathbb{A}_{k}^{1}$ under $G$, and consider the twisted group $E_{0} G$ which, for convenience, we will denote by $G_{0}$. We have a canonical bijection (the twisting map. See [DG])

$$
\eta: H^{1}\left(\mathbb{A}_{k}^{1}, G_{0}\right) \rightarrow H^{1}\left(\mathbb{A}_{k}^{1}, G\right)
$$

which maps the trivial class of $H^{1}\left(\mathbb{A}_{k}^{1}, G_{0}\right)$ to $\left[E_{0}\right]$. Furthermore $\eta$ maps constant (resp. geometrically separably trivial) torsors into constant (resp. geometrically separably trivial) torsors. By replacing $G$ by $G_{0}$ we may thus assume without loss of generality that $E_{0}$ is trivial. 
Let $C=G / G^{\circ}$. This is a twisted constant group ([DG] II §5). Consider the commutative diagram

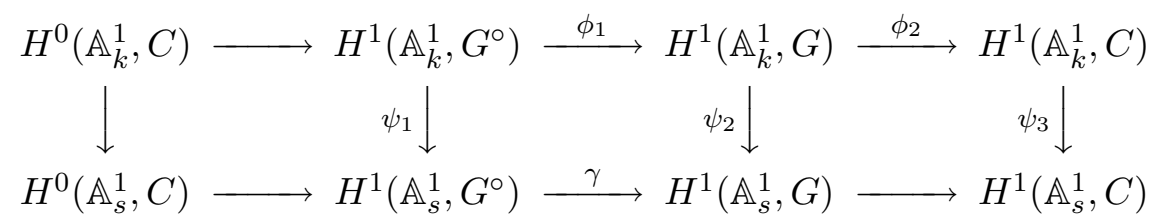

Since by assumption $\psi_{2}([E])=1$, we have $\psi_{3}\left(\phi_{2}([E])\right)=1$. By Remark 3.2 we have $\phi_{2}([E]) \in H^{1}\left(\operatorname{Gal}\left(k_{s} / k\right), C\left(\mathbb{A}_{s}^{1}\right)\right) \subset H^{1}\left(\mathbb{A}_{s}^{1}, C\right)$. But since $C_{s}$ is a constant group $C\left(\mathbb{A}_{s}^{1}\right)=C\left(k_{s}\right)$. Thus

$$
H^{1}\left(\operatorname{Gal}\left(k_{s} / k\right), C\left(\mathbb{A}_{s}^{1}\right)\right)=H^{1}\left(\operatorname{Gal}\left(k_{s} / k\right), C\left(k_{s}\right)\right) .
$$

In other words, $\phi_{2}([E])$ is a constant class, hence trivial because of our assumption on the fiber $E_{0}$ of $E$.

Let $E^{\circ}$ be an $\mathbb{A}_{k}^{1}$-torsor under $G^{\circ}$ such that $\left[E^{\circ}\right] \in H^{1}\left(\mathbb{A}_{k}^{1}, G^{\circ}\right)$ satisfies $\phi_{1}\left(\left[E^{\circ}\right]\right)=[E]$. It remains to show that $\psi_{1}\left(\left[E^{\circ}\right]\right)=1$. As before, it suffices to prove that $\gamma$ has trivial kernel or, equivalently, that $G\left(\mathbb{A}_{s}^{1}\right) \rightarrow C\left(\mathbb{A}_{s}^{1}\right)$ is surjective. But this is clear. Indeed, we have already observed above that $C\left(\mathbb{A}_{s}^{1}\right)=C\left(k_{s}\right)$.

We now turn to the proof of Theorem 2.1. By Proposition 4.3 we may assume that $G$ is reductive. Let ${ }_{E} G$ be the corresponding twisted $\mathbb{A}_{k}^{1}$-group. Let $k^{\prime} \subset k_{s}$ be a finite Galois extension such that $G_{k^{\prime}}$ is split and $E_{\mathbb{A}_{k^{\prime}}^{1}}$ is trivial. By the Lemmata of $\S 3$ applied to $Y=\mathbb{A}_{k^{\prime}}^{1}$ and Lemma 4.1 it will suffice to show that the extension $k^{\prime}$ above can be chosen so that

(a) There exists a maximal torus $T^{\prime}$ of ${ }_{E} G$ and a maximal torus $T$ of $G$ such that $T_{Y}^{\prime}$ is conjugate to $T_{Y}=T \times_{k} Y$ under $G(Y)$.

Let $\widetilde{G} \rightarrow G$ be the simply connected cover of the derived group of $G$. We can then construct the twisted group ${ }_{E} \widetilde{G}$ by considering the adjoint action of $G$ on $\widetilde{G}$, and this coincides with the simply connected cover of the derived group of the twisted group ${ }_{E} G$ [SGA3]. There exists a natural correspondence between the maximal tori of ${ }_{E} \widetilde{G}$ and those of ${ }_{E} G$ (ibid.) This shows that in order to establish that (a) above we may (and henceforth do) assume that $G$ is simply connected.

By choosing a point $p$ in the building $\mathcal{B}$ of ${ }_{E} G_{\widehat{K}}$ we obtain a group $H(p)$ over $\mathbb{P}_{k}^{1}$ extending ${ }_{E} G$. Let $S$ be an algebraic $k$-scheme representing the Weil restriction of $H(p)$ to $k$, and $S_{r}$ its corresponding separably reduced version. We have the evaluation maps

$$
e v_{\alpha}^{r}: S_{r} \times_{k} \mathbb{P}_{k}^{1} \hookrightarrow S \times_{k} \mathbb{P}_{k}^{1} \stackrel{e v_{\alpha}}{\longrightarrow} H(p) .
$$

Since $e v_{\alpha}: S \times_{k} \mathbb{P}_{k}^{1} \rightarrow H(p)$ is a morphism over $\mathbb{P}_{k}^{1}$ we see that the restriction of $e v_{\alpha}$ to $S \times_{k} \mathbb{A}_{k}^{1}$ maps into ${ }_{E} G$. This gives the commutative 
diagram

$$
\begin{array}{cc}
S_{r} \times \mathbb{P}_{k}^{1} \stackrel{e v_{\alpha}^{r}}{\longrightarrow} H(p) \\
\cup & \\
S_{r} \times \mathbb{A}_{k}^{1} \stackrel{e v_{\alpha}^{r}}{\longrightarrow} & { }_{E} G
\end{array}
$$

By Proposition 3.34.1 we obtain

(b) If $T_{r}$ is a maximal torus of $S_{r}$ (which exists since $S_{r}$ is an algebraic group over $k$ ) then $T^{\prime}=e v_{\alpha}^{r}\left(T_{r} \times \mathbb{A}_{k}^{1}\right)$ is a torus of ${ }_{E} G$.

Since the evaluation map commutes with arbitrary base field change (Remark 3.31) and the construction of $S_{r}$ commutes with any separable field extension $k^{\prime} / k$ base change (Appendix, Proposition 6.12) we may replace $k$ by $k^{\prime}$ when trying to show that the torus $T^{\prime}$ of (b) satisfies the conditions of (a). Indeed, if $\alpha^{\prime}: h_{S^{\prime}} \rightarrow \prod_{X^{\prime} / k^{\prime}}\left(H(p) \times_{k} k^{\prime}\right)$ is as in Remark 3.31, then the closed immersion

$$
\left(T_{r} \times_{k} \mathbb{A}_{k}^{1}\right) \times_{k} k^{\prime} \simeq\left(T_{r} \times_{k} k^{\prime}\right) \times_{k^{\prime}} \mathbb{A}_{k^{\prime}}^{1} \stackrel{e v_{\alpha^{\prime}}^{r}}{\longrightarrow} G \times_{k} k^{\prime}
$$

makes $T^{\prime} \times{ }_{k} k^{\prime} \simeq T^{\prime} \times{ }_{\mathbb{A}_{k}^{1}} \mathbb{A}_{k^{\prime}}^{1}$ into a torus of ${ }_{E} G \times \times_{k} k^{\prime} \simeq{ }_{E} G \times_{\mathbb{A}_{k}^{1}} \mathbb{A}_{k^{\prime}}^{1}$. If this last torus is maximal, then so is our original $T^{\prime}$ (since the maximality at the level of the geometric fibers is preserved under our base change).

We may therefore assume that ${ }_{E} G=G \times_{k} \mathbb{A}_{k}^{1}=G_{\mathbb{A}_{k}^{1}}$. Let $T$ be a maximal split torus of $G$. By Soulé's theorem [So] there exists $g \in G(k[t])$ such that $q=g(p)$ is a point in the apartment of $\mathcal{B}_{\widehat{K}}$ corresponding to $T_{\widehat{K}}$. As explained in Example 3.21 (d) we have an isomorphism $\tilde{g}: H(p) \rightarrow H(q)$ of $\mathbb{P}_{k}^{1}$-schemes. The pullback of $\tilde{g}$ along $\mathbb{A}_{k}^{1} \subset \mathbb{P}_{k}^{1}$ is an inner automorphism of $G_{\mathbb{A}_{k}^{1}}$. Thus, by replacing $T^{\prime}$ by $\tilde{g}\left(T^{\prime}\right)$ and by taking Remark 3.21(b) into consideration we may assume without loss of generality that $p=q$,

By Example 3.21 (c) the torus $T$ yields a closed immersion

$$
T \times_{k} \mathbb{P}_{k}^{1} \longrightarrow H(q)
$$

which by Remark 3.32 is nothing but the restriction of the reduced evaluation map, namely

$$
\mathrm{ev}_{\alpha}^{r}: T \times_{k} \mathbb{P}_{k}^{1} \rightarrow S_{r} \times_{k} \mathbb{P}_{k}^{1} \rightarrow S \times_{k} \mathbb{P}_{k}^{1} \stackrel{e v_{\alpha}}{\rightarrow} H(q) .
$$

By pulling back along $\mathbb{A}_{k}^{1}$ we see that the reduced evaluation map induces a closed immersion

$$
e v_{\alpha}^{r}: T \times_{k} \mathbb{A}_{k}^{1} \longrightarrow G_{\mathbb{A}_{k}^{1}}
$$

By Remark 3.30 we know that the functor of global sections of $T \times_{k} \mathbb{P}_{k}^{1}$ is represented by $T$. Thus from the closed immersion (4.4) we obtain a canonical embedding $T \rightarrow S$ (see Remark 3.32) which factors through $S_{r}$ since $T$ is reduced. The resulting map $T \rightarrow S_{r}$ is an injective morphism of algebraic groups, hence a closed embedding. This allows us to identify $T$ 
with a torus of $S_{r}$. By Proposition 3.34 this torus is necessarily maximal. We record this important fact for future reference.

(c) If $T_{r}$ is a maximal torus of $S_{r}$ then $T^{\prime}=e v_{\alpha}^{r}\left(T_{r} \times \mathbb{A}_{k}^{1}\right)$ is a maximal torus of ${ }_{E} G$. In particular the $\mathbb{A}_{k}^{1}$-group ${ }_{E} G$ admits a maximal torus.

We will see that $T^{\prime}$ actually satisfies the conditions of (a). This will finish the proof of Theorem 2.1

Let $k^{\prime} \subset k_{s}$ be a finite extension of $k$ such that $T_{r} \times_{k} k^{\prime}$ and $T \times_{k} k^{\prime}$ are conjugate under an element $s \in S_{r}\left(k^{\prime}\right) \subset S\left(k^{\prime}\right)$. We again replace $k$ by $k^{\prime}$. Think of $s$ as an $\mathbb{A}_{k}^{1}$-point of $S \times{ }_{k} \mathbb{A}_{k}^{1}$ (which we denote by $\tilde{s}$ ). Then the inner automorphism $\operatorname{int}(\tilde{s})$ of $S \times{ }_{k} \mathbb{A}_{k}^{1}$ is given by $\operatorname{int}(s) \times i d$. Consider the element

$$
e v_{\alpha}(\tilde{s}) \in G_{\mathbb{A}_{k}^{1}}\left(\mathbb{A}_{k}^{1}\right)=G\left(\mathbb{A}_{k}^{1}\right) .
$$

We have the commutative diagram

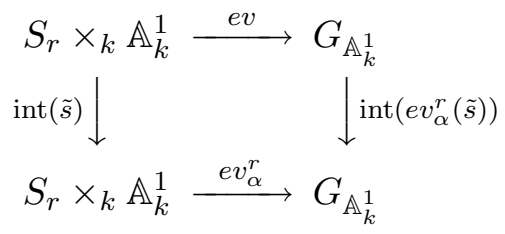

Since $\operatorname{int}(s)\left(T_{r}\right)=T$ this last diagram shows that $T^{\prime}=\operatorname{ev}_{\alpha}^{r}\left(T_{r} \times_{k} \mathbb{A}_{k}^{1}\right)$ is conjugate to $e v_{r}\left(T \times_{k} \mathbb{A}_{k}^{1}\right)$ under the element $\operatorname{int}\left(e v_{\alpha}(\tilde{s})\right) \in G\left(\mathbb{A}_{k}^{1}\right)$. Since $e v_{\alpha}\left(T \times_{k} \mathbb{A}_{k}^{1}\right)$ is a maximal torus of $G_{\mathbb{A}_{k}^{1}}$ the proof of (a), hence also of Theorem 2.1, is complete.

By reasoning as in Steps (a), (b) and (c) of the above proof we obtain the following important fact.

4.6. Theorem. Let $G$ be a reductive group scheme over $\mathbb{A}_{k}^{1}$. Assume that $G$ is "geometrically separably split", i.e. that $G \times_{\mathbb{A}_{k}^{1}} \mathbb{A}_{k_{s}}^{1}$ is split. Then $G$ has a maximal torus.

\section{TORSORS OVER THE PUNCTURED AFFine LiNE}

In what follows we will denote by $X=\operatorname{Spec} k\left[t^{ \pm 1}\right]$ the punctured affine line and by $K=k(t)$ the rational function field of $X$. We will denote by $\widetilde{K}$ the maximal unramified extension of $\widehat{K}=k((t))$. Recall that $\widetilde{K}$ is the subfield of $k_{s}((t))$ consisting of those elements $f=\sum_{n \geq N} c_{n} t^{n}$ for which the set $\left\{c_{n}: n \geq N\right\}$ belong to a finite separable extension of $k$. The natural map $\operatorname{Gal}\left(k_{s} / k\right) \rightarrow \operatorname{Gal}(\widetilde{K} / \widehat{K})$ is an isomorphism of profinite groups. We henceforth identify these two groups.

Let $\widehat{O}=k[[t]]$ and $\widetilde{O}=k_{s}[[t]] \cap \widetilde{K}$. The residue map $\rho: \widetilde{O} \rightarrow k_{s}$ (which is also the specialization map at $t=0$ ) induces a group homomorphism $G(\rho): G(\widetilde{O}) \rightarrow G\left(k_{s}\right)$ whose kernel we denote by $G(\widetilde{O})^{(1)}:$ These are "the elements of $G(\widetilde{O})$ that are congruent to 1 modulo $(t)$ ". We denote $\mathrm{G}_{m}(\widetilde{O})^{(1)}$ simply by $\widetilde{O}^{(1)}$. Thus $O^{(1)}=\left\{\sum_{n \geq 0} c_{n} t^{n} \in \widetilde{O}: c_{0}=1\right\}$. 
Our goal is to prove Theorem 2.4 by comparing the étale cohomology of $X$ with the Galois cohomology of $k((t))$. As in the case of the affine line, the existence of maximal tori will play a crucial role.

5.1. Cohomological exponent: Good and bad primes. Theorem 2.4 for the punctured line has an assumption on the "good characteristic" on the base field. In this section we give the relevant definitions and basic results concerning this point.

5.1. Lemma. Let $G$ be a reductive $k$-group. There exists a positive integer $n$ with the property that for every field extension $F / k$ and for every maximal torus $T$ of the reductive $F$-group $G_{F}$

(1) $n H^{1}(F, T)=1$.

Moreover, if $X(T)_{*}$ denotes the $\operatorname{Gal}\left(F_{\mathrm{sep}} / F\right)$-module of cocharacters of $T$ (see 3.1), then

(2) $n H^{1}\left(F, X(T)_{*}\right)=1$.

Proof. (1) Let $T$ be a maximal torus of $G_{F}$. Consider a minimal splitting extension $L / F$ of $T$. Since $H^{1}(L, T)=1$ we have $H^{1}(F, T)=H^{1}(L / F, T(L))$. In particular, if $m=[L: F]$ then $m H^{1}(F, T)=1$. We now show that there is a positive integer $n$ which does not depend on $T$ and $F$ such that $m$ divides $n$. This would complete the proof of (a).

Recall that $G$ is the almost direct product $G=C \cdot G^{\prime}$ of its central torus $C$ and derived group $G^{\prime}$. This yields $T=C_{F} \cdot T^{\prime}$ where $T^{\prime}$ is the maximal torus $G_{F}^{\prime}$ given by $T^{\prime}=G_{F}^{\prime} \cap T$.

Let $l / k$ be the minimal field extension of $k$ splitting $C$. The decomposition $T=C_{F} \cdot T^{\prime}$ implies that $L$ is the composition $L=L_{1} \cdot L_{2}$ (taken inside some fixed separable closure $F_{s}$ of $F$ containing $k_{s}$ ) of two fields $L_{1}$ and $L_{2}$ where $L_{1}=l \cdot F$ and $L_{2}$ is the minimal splitting extension of the $F$-torus $T^{\prime}$. It is well known that $\operatorname{Gal}\left(L_{2} / F\right)$ admits an embedding into the automorphism group Aut $(\Sigma)$ of the root system $\Sigma=\Sigma\left(G_{F}^{\prime}, T^{\prime}\right)$ of $G_{F}^{\prime}$ with respect to $T^{\prime}$. It is easy to see that the positive integer $n=[l: k] \cdot \mid$ Aut $(\Sigma) \mid$ is then divisible by $m=[L: F]$. Observe that $n$ depends neither on $T$ nor $F$.

(2) Let $T$ be a maximal $F$-torus of $G_{F}$. As will be explained in Lemma 5.16(3) $X(T)_{*}$ is a Galois stable direct summand of $T(\widetilde{F(t)})$. From this it follows that $H^{1}\left(F, X(T)_{*}\right)$ is a direct summand of $H^{1}\left(\operatorname{Gal}\left(F_{\text {sep }} / F\right), T(\widetilde{F(t)}) \subset\right.$ $H^{1}(\widehat{F(t)}, T)$, hence that $n H^{1}\left(F, X(T)_{*}\right)=0 .{ }^{5}$

Let $G$ be a reductive $k$-group. The smallest positive integer satisfying the conditions of Lemma 5.1 is called the cohomological toral exponent of $G$. It will be denoted by $\operatorname{cte}(G)$.

We will use cte $(G)$ to define the concept of good and bad primes for $G$. We first define the relevant concepts in the semisimple case. Let $G$ be a

\footnotetext{
${ }^{5}$ Since $T$ splits over $F_{\text {sep }}$ the inclusion $H^{1}\left(\operatorname{Gal}\left(F_{\text {sep }} / F\right), T(\widetilde{F(t)}) \subset H^{1}(\widehat{F(t)}, T)\right.$ is in fact an equality.
} 
semisimple $k$-group. There exists a unique Chevalley form of $G$, that is a Chevalley group $H=H_{G}$ such that $H \otimes_{\mathbb{Z}} k_{s} \simeq G \otimes_{k} k_{s}$. Recall [SGA3] Exp. XXIV.3 that we have a split exact sequence of $\mathbb{Z}$-groups

$$
1 \rightarrow H_{\mathrm{ad}} \rightarrow \operatorname{Aut}(H) \rightarrow \operatorname{Out}(H) \rightarrow 1,
$$

where $H_{\text {ad }}$ is the adjoint group of $H$ and Out $(G)$ is the "group of outer automorphisms" of $H$. The group $\operatorname{Out}(H)$ is a finite constant $\mathbb{Z}$-group whose underlying abstract group is a subgroup of the group of symmetries of the Dynkin diagram of $H$. The sequence (5.2) comes equipped with a natural section $\operatorname{Out}(H) \rightarrow \operatorname{Aut}(H)$ that arises from the fixed choice of "épinglage" used in defining $H$. After applying the base change $\mathbb{Z} \rightarrow k$ and passing to cohomology (5.2) yields the exact sequence of pointed sets

$$
H^{1}\left(k, H_{\mathrm{ad}}\right) \rightarrow H^{1}(k, \operatorname{Aut}(H)) \rightarrow H^{1}(k, \operatorname{Out}(H)) \rightarrow 1 .
$$

Let $z \in Z^{1}(k, \operatorname{Aut}(H))$ be such that $G \simeq{ }_{z} H$. Let $\left[z^{\prime}\right]$ be the image of $[z]$ under the morphism $H^{1}(k, \operatorname{Aut}(H)) \rightarrow H^{1}(k, \operatorname{Out}(H))$. There is a canonical section $H^{1}(k, \operatorname{Out}(H)) \rightarrow H^{1}(k, \operatorname{Aut}(H))$ which is obtained from the given section in (5.2). Let $\left[z^{\prime \prime}\right]$ be the image of $\left[z^{\prime}\right]$ with respect to this last mapping. Clearly, the twisted group $G_{\mathrm{qs}}={ }_{z^{\prime \prime}} H_{k}$ is quasi-split. We call $G_{\mathrm{qs}}$ the (Chevalley) quasi-split form of $G$. It has the following characteristic properties:

(a) $G_{\mathrm{qs}}$ is a $k$-form of $G$, i.e. $G_{\mathrm{qs}}$ and $G$ are isomorphic over $k_{s}$;

(b) for a field extension $F / k$ the group $G_{F}$ is an inner form of $H_{F}$ if and only if $G_{\mathrm{qs}} \times{ }_{k} F$ is split. In particular, the star-action of $\operatorname{Gal}\left(k_{s} / k\right)$ on the Dynkin diagrams of $G_{\mathrm{qs}}$ and $G$ is the same.

5.4. Lemma. If $P$ is a parabolic subgroup of $G$, then $G_{\mathrm{qs}}$ contains a parabolic subgroup of type $\mathbf{t}(P)$.

Proof. Let $\mathbf{t}$ denote the type of $P$. Since $P$ is $k$-defined the quotient variety $G / P$ is $k$-defined as well. In particular $\mathbf{t}$ is stable with respect to the star action of $\operatorname{Gal}\left(k_{s} / k\right)$ on the Dynkin diagram. Let $Q$ be a parabolic subgroup of the $k_{s}$ reductive group $G_{\mathrm{qs}_{s}}=G_{\mathrm{qs}} \times_{k} k_{s}$ of type $\mathbf{t}$. Since $\mathbf{t}$ is Galois stable, the variety $G_{\mathrm{qs}_{s}} / Q$ is $k$-defined, hence isomorphic to the variety $\operatorname{Par}_{\mathbf{t}}\left(G_{\mathrm{qS}}\right)$ of parabolic subgroups in $G_{\mathrm{qs}}$ of type $\mathbf{t}$ (c.f. [MPW, prop. 1.3]).

Analogously, if $B$ is a Borel subgroup of $G_{\mathrm{qs}}$ (which exists, since $G_{\mathrm{qs}}$ is quasisplit) the variety $G_{\mathrm{qs}} / B$ is isomorphic to the variety $\operatorname{Bor}\left(G_{\mathrm{qs}}\right)$ of Borel subgroups in $G_{\mathrm{qs}}$. Without loss of generality we may assume that $Q$ contains $B$. The canonical morphism $G_{\mathrm{qS}} / B \rightarrow G_{\mathrm{qs}} / Q$ is $k$-defined. Since $\left(G_{\mathrm{qS}} / B\right)(k) \neq \emptyset$ we have $\operatorname{Par}_{\mathbf{t}}\left(G_{\mathrm{qS}}\right)(k)=\left(G_{\mathrm{qS}} / Q\right)(k) \neq \emptyset$ and the Lemma follows.

Next we define the concept of quasisplit form in the reductive case. Let $G$ be a reductive $k$-group, and let $C$ denote its radical torus [SGA3, XXII.4.3.6]. 
Recall that $C$ is the unique maximal torus of the center of $G$. It is not difficult to see that up to isomorphism there exists a unique reductive $k$-group $G_{\mathrm{qs}}$, called the (Chevalley) quasisplit form of $G$, with the following two properties:

(1) The central torus of $G$ and $G_{\mathrm{qs}}$ are isomorphic.

(2) $\operatorname{Der}\left(G_{\mathrm{qs}}\right)=\operatorname{Der}(G)_{\mathrm{qs}}$. That is, the quasisplit form of the (semisimple) derived group of $G$ coincides with the derived group of $G_{\mathrm{qs}}$.

We can now state the definition of good and bad primes: Let $G$ be a reductive $k$-group and $G_{\mathrm{qs}}$ its quasisplit form. The prime divisors of the cohomological toral exponent $\operatorname{cte}\left(G_{\mathrm{qs}}\right)$ of $G_{\mathrm{qs}}$ are called bad primes for $G$. Prime numbers which are not bad are called good. We say that the characteristic $p$ of the base field $k$ is good for $G$ if either $p=0$ or $p$ is a good prime for $G$.

5.5. Remark. From Steinberg's work we know that every maximal $k$-torus of $G$ admits a $k$-embedding into $G_{\mathrm{qs}}$. From the definition it follows that cte $(\mathrm{G})$ divides $\operatorname{cte}\left(G_{\mathrm{qS}}\right)$. In particular the characteristic of $k$ does not divide $\operatorname{cte}(\mathrm{G})$.

5.6. Remark. The cohomological toral exponent "depends on the base field". If $G$ is a trialitarian $k$-group, then 3 divides cte $(G)$ but not $\operatorname{cte}\left(G_{k_{s}}\right)$ (this last since $G_{k_{s}}$ is a classical group of type $D_{4}$ ).

We now state and prove two stability properties of the set of good primes which will be used while proving the main result on torsors over the punctured line.

5.7. Lemma. Let $G$ be a reductive $k$-group. Let $P \subset G$ be a parabolic subgroup. If $H$ is a Levi subgroup of $P$ then the good primes for $G$ are also good primes for $H$. In particular, if the characteristic of $k$ is good for $G$, then it is also good for $H$.

Proof. Let $G_{\mathrm{qs}}$ be the quasi-split form of $G$, and let $P_{0}$ be a parabolic subgroup of $G_{\mathrm{qS}}$ of the same type as $P$ (see Lemma 5.4). Clearly any Levi subgroup $H_{0}$ of $P_{0}$ is isomorphic to the quasisplit form $H_{\mathrm{qs}}$ of $H$. Since for any field extension $F / k$ any maximal torus of $H_{0, F}$ is also a maximal torus of $G_{\mathrm{qs}, F}$ we see that $\operatorname{cte}\left(H_{0}\right)$ divides $\operatorname{cte}\left(G_{\mathrm{qS}}\right)$. The result now follows.

5.8. Lemma. Let $\eta \in Z^{1}(k, G)$ be a cocycle and ${ }_{\eta} G$ the corresponding twisted group. The set of good primes for $G$ and ${ }_{\eta} G$ coincide.

Proof. Indeed, the quasisplit forms of $G$ and ${ }_{\eta} G$ are isomorphic, so the result follows by definition.

5.2. Existence of maximal tori. The following result yields the existence of maximal tori that will be used in the proof of Theorem 2.4.

5.9. Proposition. Let $G$ be a reductive group scheme over $X$. Assume that $G$ is geometrically separably split; that is there exists an isomorphism 
$f: G_{0} \times_{\mathbb{Z}} X_{k^{\prime}} \stackrel{\sim}{\longrightarrow} G \times_{X} X_{k^{\prime}}$ where $G_{0} / \mathbb{Z}$ is a Chevalley reductive group and $k^{\prime} / k$ is a finite Galois extension.

Denote by $T_{0}$ the standard maximal split torus of the $\mathbb{Z}$-group $G_{0}$. Then $G$ admits a maximal $X$-torus $M$ such that $M \times_{X} X_{s}$ is $G\left(X_{s}\right)$-conjugated to $f\left(T_{0} \times_{\mathbb{Z}} X_{s}\right)$.

Proof. By [SGA3, XII.4.7.c] there is a one-to-one correspondence between the maximal tori of $G$, those of its adjoint group $G_{\text {ad }}$ and those of the simply connected covering of $G_{\text {ad }}$. We may thus assume without loss of generality that our $X$-group $G$ is semisimple and simply connected. Following Tits [Ti3], we consider the twin building $\mathcal{B}=\mathcal{B}_{+} \times \mathcal{B}_{-}$of $G_{K}$ over the completions of $K$ at 0 and $\infty$.

Consider a point $p=\left(p_{+}, p_{-}\right) \in \mathcal{B}$, as well as the two associated parahoric group schemes $F_{+} / k[[t]]$ and $F_{-} / k\left[\left[\frac{1}{t}\right]\right]$ corresponding to $p_{+}$and $p_{-}$respectively. The patching process of $\S 5$ (applied twice) produces a smooth group scheme $H(p)$ over $\mathbb{P}_{k}^{1}$ extending $G / X$. Let $S$ be the $k$-group representing the Weil restriction of $H(p)$ to $k$, and $S_{r}$ the corresponding absolutely reduced group.

Let $T_{r} \subset S_{r}$ be a maximal torus. By Proposition 3.34.1 ev $\left(T_{r} \times_{k} \mathbb{P}_{k}\right)$ is a torus of $H(p)$. Pulling back to $X$ we obtain that $M=e v\left(T_{r} \times_{k} X\right)$ is a torus of $G$. We will show that $M$ has the desired properties. The same reasoning given for the affine shows that we may replace $k$ by $k^{\prime}$.

There is a canonical embedding $\mathcal{B} \rightarrow \mathcal{B}^{\prime}$ where $\mathcal{B}^{\prime}$ is the twin building associated to $G \times_{k((t))} k^{\prime}((t))$ and $G \times_{k((t))} k^{\prime}\left(\left(\frac{1}{t}\right)\right)$. This allows us to view our chosen point $p$ as an element of the twin building $\mathcal{B}^{\prime}$. The construction of $H(p)$ is compatible with this identification [see Example 3.21(b)].

We now use the splitting $G_{0} \times_{k} X_{k^{\prime}} \stackrel{\sim}{\longrightarrow} G \times_{X} X_{k^{\prime}}$. By Abramenko's result [A, Proposition 5], there exists $g \in G\left(X_{k^{\prime}}\right)$ such that $q=g(p)$ lives in the canonical twin apartment corresponding to the torus $T_{0}$. Clearly the group schemes $H(q)$ and $H(p) \times_{\mathbb{P}^{1}} \mathbb{P}_{k^{\prime}}^{1}$ are isomorphic, so we may assume that $p=q$. The torus $T_{0}$ gives rise to a canonical subtorus $T_{0} \times_{\mathbb{Z}} \mathbb{P}_{k^{\prime}}^{1} \subset H(p)$, as one can see by applying the reasoning of Example 3.21(c) twice.

The proof can now be finished along the exact same reasoning given in the proof of the affine line.

5.3. Reformulation of Theorem 2.4. Henceforth $G$ will denote a reductive $k$-group where the characteristic of $k$ is good for $G$.

By Remark 2.1 the isomorphism classes of geometrically separably split torsors over $\mathbb{A}_{k}^{\times}$under $G$ are parametrized by $H^{1}\left(\operatorname{Gal}\left(k_{s} / k\right), G\left(k_{s}\left[t^{ \pm 1}\right]\right)\right)$. Along similar lines we see that the classes of $H^{1}(\widehat{K}, G)$ corresponding to torsors that are trivialized by the base change $\widehat{K} \rightarrow \widetilde{K}$ are parametrized by $H^{1}(\operatorname{Gal}(\widetilde{K} / \widehat{K}), G(\widetilde{K}))$. Theorem 2.4 can thus be stated as follows:

5.10. Proposition. Under the natural identification of $\Gamma:=\operatorname{Gal}\left(k_{s} / k\right)$ with $\operatorname{Gal}(\widetilde{K} / \widehat{K})$ we have. 
(1) Every $X$-torsor under $G$ is geometrically separably trivial. In particular the canonical map $H^{1}\left(\Gamma, G\left(k_{s}\left[t^{ \pm 1}\right]\right)\right) \rightarrow H^{1}(X, G)$ is bijective.

(2) The canonical map $H^{1}(\Gamma, G(\widetilde{K})) \rightarrow H^{1}(\widehat{K}, G)$ is bijective: Every $\widehat{K}$ torsor under $G$ is split by the base change $\widehat{K} \rightarrow \widetilde{K}$.

(3) The thesis of Theorem 2.4 is equivalent to the following assertion: The canonical map

is bijective.

$$
H^{1}\left(\Gamma, G\left(k_{s}\left[t^{ \pm 1}\right]\right)\right) \rightarrow H^{1}(\Gamma, G(\widetilde{K}))
$$

We begin with a useful general fact.

5.11. Lemma. Let $G$ be a split reductive group over a field $F$. Let $z \in$ $Z^{1}(F, G)$ be a cocycle. There exists a maximal torus $T \subset G$ such that $[z]$ is in the image of the natural map $H^{1}(F, T) \rightarrow H^{1}(F, G)$.

Proof. The result essentially follows from arguments in Steinberg's paper [St], which show that the Lemma holds for quasi-split simple group (see the prelude to Theorem 3.1 of [Chr] for details). In particular, the Lemma holds if our $G$ is semisimple and of adjoint type. The reduction to this case is done along standard lines as follows.

Let $C$ be the centre of $G$ and let $G^{\prime}=G / C$. Let $z^{\prime} \in Z^{1}\left(F, G^{\prime}\right)$ be the image of $z$ under the canonical map $Z^{1}(F, G) \rightarrow Z^{1}\left(F, G^{\prime}\right)$. Since $G^{\prime}$ is an adjoint group there exists a maximal torus $T^{\prime} \subset G^{\prime}$ such that $\left[z^{\prime}\right] \in$ $\operatorname{Im}\left[H^{1}\left(F, T^{\prime}\right) \rightarrow H^{1}\left(F, G^{\prime}\right)\right]$. Let $T \subset G$ be the inverse image of $T^{\prime}$ under $G \rightarrow G^{\prime}$. Since the image of $\left[z^{\prime}\right]$ under the map $H^{1}\left(F, G^{\prime}\right) \rightarrow H^{2}(F, C)$ vanishes, the class $\left[z^{\prime}\right]$ lifts to a class $[u] \in H^{1}(F, T)$ as one can see by considering the exact sequence $1 \rightarrow C \rightarrow T \rightarrow T^{\prime} \rightarrow 1$. We now pass to the twisted group ${ }_{u} G$. Under the twisting bijection $H^{1}(F, G) \rightarrow H^{1}\left(F,{ }_{u} G\right)$ the class $[z]$ goes into some class, say $[w]$, whose image under $H^{1}\left(F,{ }_{u} G\right) \rightarrow$ $H^{1}\left(F,{ }_{u} G^{\prime}\right)$ is zero. Hence we may assume that $[w] \in H^{1}\left(F,{ }_{u} C\right)$. Note that ${ }_{u} C=C$ is the centre of ${ }_{u} G$ and is contained in ${ }_{u} T=T$. It follows that $[z]=[u+w]$ where the sum $[u+w]=[u]+[w]$ is taken inside the group $H^{1}(F, T)$.

We now turn to the proof of Proposition 5.10. We begin by showing that

$$
H^{1}\left(k_{s}(t), G\right)=1 .
$$

For convenience we denote $k_{s}(t)$ by $F$. The main fundamental property of $F$ we are going to use is that it is a field of $q$-cohomological dimension 1 for all primes $q$ different than the characteristic $p$ of $k$ [Se1, §II.4.2].

Let $z \in Z^{1}(F, G)$ be a cocycle. Since $G_{F}$ is split, the previous Lemma reduces the problem to showing that $H^{1}(F, T)=1$ for any maximal torus $T$ of $G_{F}$.

Let $L / F$ be a minimal Galois extension splitting $T$. Since $T$ is split over $L$ we have $H^{1}(L, T)=1$, hence $H^{1}(L / F, T(L))=H^{1}(F, T)$. Because of the definition of good characteristic the abelian group $H^{1}(L / K, T(L))$ is the 
direct sum of its $q$-Sylow subgroups $H^{1}(L / F, T(L))_{q}$ where $q$ runs through the set of primes other than $\mathrm{p}$ that divide $n=[L: F]$.

Let $\Gamma$ be the Galois group of $L / F$. For each prime $q$ dividing the order of $\Gamma$ we fix a $q$-Sylow subgroup $\Gamma_{q} \subset \Gamma$. We have the tower of fields $F \subset L_{q} \subset L$ where $L_{q}$ is the subfield in $L$ corresponding to $\Gamma_{q}$. The standard restrictioncorestriction argument shows that the equality $H^{1}(L / F, T(L))_{q}=1$ follows immediately from that of $H^{1}\left(L / L_{q}, T(L)\right)=1$, or equivalently, from $H^{1}\left(L_{q}, T\right)=1$. To establish this last equality we consider an exact sequence of $L_{q}$-tori

$$
1 \rightarrow T_{1} \rightarrow P \rightarrow T \rightarrow 1
$$

where $P$ is a permutation torus ([CTS2] lemme 3 ), and its corresponding Galois cohomology sequence. From this it follows that it will suffice to show that the $q$-torsion part of $H^{2}\left(L_{q}, T_{1}\right)$ is trivial. Let $A \subset T_{1}$ be the $q^{m}$-torsion part of $T_{1}$ where $m$ is suitably large. Every element in $H^{2}\left(L_{q}, T_{1}\right)$ of order a power of $q$ comes from $H^{2}\left(L_{q}, A\right)$. Since $L_{q}$ is of $q$-cohomological dimension 1 we have $H^{2}\left(L_{q}, A\right)=1$. This finishes the proof of (5.12).

We now turn to the proof of (1). Let $E$ be a $G$-torsor over $X_{s}$ and let ${ }_{E} G$ be the corresponding twisted $X_{s}$-group scheme. The idea of the proof is to show that $E$ admits a reduction of structure group to a Borel subgroup $B$ of $G$. In other words, we want to prove that the isomorphism class of $E$ is in the image of

$$
H^{1}\left(X_{s}, B\right) \rightarrow H^{1}\left(X_{s}, G\right) .
$$

Recall that the class of $E$ is in the image of the map (5.13) if and only if the $X_{s}$-group scheme ${ }_{E} G$ has a Borel subgroup.

By (5.12) the generic fiber of ${ }_{E} G$ is split. In particular this generic fiber has a Borel subgroup over $k_{s}(t)$. Since $X_{s}=\operatorname{Spec}\left(k_{s}\left[t^{ \pm 1}\right]\right)$ has dimension 1 , it follows from a standard argument (see Remark 5.31 below) that ${ }_{E} G$ itself has a Borel subgroup as required. It remains to show that $H^{1}\left(X_{s}, B\right)=1$, but this is clear by devissage since $H^{1}\left(X_{s}, \mathrm{G}_{a}\right)=1$ and the Picard group of $X_{s}$ is trivial.

(2) In analogy to (5.12) we have

$$
H^{1}\left(\widetilde{k_{s}(t)}, G\right)=1 \text {. }
$$

This follows by reasoning as in (1) by taking into consideration that $\widetilde{k_{s}(t)}$ is of $q$-cohomological dimension 1 for all primes $q$ other than $p$ (see [Se2] theorem 4.4). The same devissage reasoning used in (1) completes the proof.

(3) This is a direct consequence of (1) and (2).

5.4. Reduction of structure group to $N_{G}(T)$. Let $T$ be a maximal torus of our reductive $k$-group $G$. By combining Lemma 3.15 and Proposition 5.9 we obtain.

5.15. Lemma. The map

$$
H^{1}\left(\Gamma, N_{G}(T)\left(k_{s}\left[t^{ \pm 1}\right]\right)\right) \longrightarrow H^{1}\left(\Gamma, G\left(k_{s}\left[t^{ \pm 1}\right]\right)\right)
$$


is surjective.

We now look in detail at $H^{1}\left(\Gamma, N_{G}(T)\left(k_{s}\left[t^{ \pm 1}\right]\right)\right.$. Let $X(T)_{*}$ be the group of cocharacters of $T$. Recall that $X(T)_{*}$ comes equipped with a natural $\mathrm{Gal}\left(k_{s} / k\right)$-module structure (see 3.1). Since the underlying scheme of $\mathrm{G}_{m}$ is $X=\operatorname{Spec}\left(k\left[t^{ \pm 1}\right]\right)$, a cocharacter of $T$ is naturally an element of $T\left(k_{s}\left[t^{ \pm 1}\right]\right)$. This allows us to henceforth identify $X(T)_{*}$ with a Galois submodule of $T\left(k_{s}\left[t^{ \pm 1}\right]\right)$.

5.16. Lemma. Let $N=N_{G}(T)$. There exists natural Galois modules isomorphisms:

(1) $T\left(k_{s}\right) \times X(T)_{*} \stackrel{\sim}{\longrightarrow} T\left(k_{s}\left[t^{ \pm 1}\right]\right)$;

(2) $T(\widetilde{O})=T\left(k_{s}\right) \times T(\widetilde{O})^{(1)}$;

(3) $T\left(k_{s}\right) \times T(\widetilde{O})^{(1)} \times X(T)_{*} \stackrel{\sim}{\longrightarrow} T(\widetilde{K})$;

(4) $X(T)_{*} \rtimes N\left(k_{s}\right) \stackrel{\sim}{\longrightarrow} N\left(k_{s}\left[t^{ \pm 1}\right]\right)$;

(5) $X(T)_{*} \times T(\widetilde{O})^{(1)} \rtimes N\left(k_{s}\right) \stackrel{\sim}{\longrightarrow} N(\widetilde{K})$.

Proof. (1) $T_{s}$ corresponds to the ring $k_{s}\left[x_{1}^{ \pm 1}, \ldots, x_{l}^{ \pm 1}\right]$ with a given action of $\Gamma$. An element $a \in T\left(k_{s}\left[t^{ \pm 1}\right]\right)$ corresponds to a $k_{s}$-algebra homomorphism

$$
a^{*}: k_{s}\left[x_{1}^{ \pm 1}, \ldots, x_{l}^{ \pm 1}\right] \longrightarrow k_{s}\left[t^{ \pm 1}\right]
$$

which is entirely defined by the values $a^{*}\left(x_{i}\right)$. We have $a^{*}\left(x_{i}\right)=\lambda_{i} t^{n_{i}}$. It is clear that there exists $\chi \in X(T)_{*}$ such that $\chi: x_{i} \rightarrow t^{n_{i}}$. This shows that $T\left(k_{s}\left[t^{ \pm 1}\right]\right)$ is generated by $T\left(k_{s}\right)$ and $X(T)_{*}$. That the natural map $T\left(k_{s}\right) \times X(T)_{*} \rightarrow T\left(k_{s}\left[t^{ \pm 1}\right]\right)$ is injective and compatible with the action of $\Gamma$ is clear.

(2) and (3) Every element of $\widetilde{K}^{\times}$can uniquely be written in the form $\lambda f t^{n}$ with $\lambda \in k_{s}^{\times}, f \in \mathrm{G}_{m}(\widetilde{O})^{(1)}=\widetilde{O}^{(1)}$ and $n \in \mathbb{Z}$. Moreover, if the element is in $\widetilde{O}$ then $n=0$. One now reasons mutatis mutandis as in (1).

(4) We know that

$$
N_{s}=\coprod_{\bar{w} \in W} w T_{s}
$$

where the $w \in N\left(k_{s}\right)$ are representatives of the elements of the Weyl group $W$. Since $\operatorname{Spec}\left(k_{s}\left[t^{ \pm 1}\right]\right)$ is connected it is then clear that

$$
N\left(k_{s}\left[t^{ \pm 1}\right]\right)=\coprod w T\left(k_{s}\left[t^{ \pm 1}\right]\right) .
$$

If $y \in w T\left(k_{s}\left[t^{ \pm 1}\right]\right) \subset N\left(k_{s}\left[t^{ \pm 1}\right]\right)$ then

$$
w^{-1} y \in T\left(k_{s}\left[t^{ \pm 1}\right]\right)=T\left(k_{s}\right) \times X(T)_{*} .
$$

Since $N\left(k_{s}\right)=\coprod w T\left(k_{s}\right)$ the result follows (after checking compatibility with the action of $\Gamma$ ).

(5) The reasoning is similar to (3) and (4) above.

5.17. Lemma. Let $\Gamma$ denote the Galois group Gal $\left(k_{s} / k\right)$. For all $i \geq 1$, we have 
(1) $H^{i}\left(\Gamma, T(\widetilde{O})^{(1)}\right)=0$;

(2) $H^{i}\left(\Gamma, T\left(k_{s}\right)\right) \stackrel{\sim}{\longrightarrow} H^{i}(\Gamma, T(\widetilde{O}))$;

(3) $H^{i}\left(\Gamma, T\left(k_{s}\left[t^{ \pm 1}\right]\right)\right) \stackrel{\sim}{\longrightarrow} H^{i}(\Gamma, T(\widetilde{K}))$.

Proof. (1)We have a natural isomorphism

$$
\widetilde{O}^{(1)} \otimes_{\mathbb{Z}} X(T)_{*} \simeq T(\widetilde{O})^{(1)}
$$

This allows us to generalize the classical proof for $T=\mathrm{G}_{m}$ (e.g. [GS, $\S 6.3])$ in an obvious way to yield $H^{i}\left(\Gamma, T(\widetilde{O})^{(1)}\right)=0$.

(2) Follows from (1) and Lemma 5.16.2

(3) Follows from (2) and Lemma 5.16.1,3

5.5. Proof of surjectivity. The following result, together with Proposition 5.10.3 and Lemma 5.15, establishes the surjectivity part of Theorem 2.4.

5.19. Proposition. In the commutative diagram

$$
\begin{aligned}
& H^{1}\left(\Gamma, N_{G}(T)\left(k_{s}\left[t^{ \pm 1}\right]\right)\right) \stackrel{\beta_{N_{G}(T)}}{\longrightarrow} H^{1}\left(\Gamma, N_{G}(T)(\widetilde{K})\right) \\
& \rho_{X_{s}} \downarrow \quad \rho_{\widetilde{K}} \downarrow \\
& H^{1}\left(\Gamma, G\left(k_{s}\left[t^{ \pm 1}\right]\right)\right) \quad \stackrel{\beta_{G}}{\longrightarrow} \quad H^{1}(\Gamma, G(\widetilde{K}))
\end{aligned}
$$

obtained by the base change and change of structure group, the maps $\rho_{X_{s}}$, $\rho_{\widetilde{K}}$ and $\beta_{N_{G}(T)}$ are surjective (and as a consequence so is $\beta_{G}$ ).

Proof. That $\rho_{X_{s}}$ is surjective follows from Lemma 5.15. The same reasoning shows that $\rho_{\widetilde{K}}$ is surjective since we know that for all $z \in Z^{1}(\Gamma, G(\widetilde{K}))$ the twisted $\widehat{K}$-group ${ }_{z} G_{\widehat{K}}$ contains a maximal $\widehat{K}$-torus which is split by $\widetilde{K}$ [BT2, cor. 5.1.12], and any two such tori are conjugate by an element of $G(\widetilde{K})$.

It remains to show that $\beta_{N_{G}(T)}$ is surjective. For convenience we will denote $N_{G}(T)$ simply by $N$. Let $z \in Z^{1}(\Gamma, N(\widetilde{K}))$. Then $z=\left(z_{\gamma}\right)_{\gamma \in \Gamma}$ for some $z_{\gamma} \in N(\widetilde{K})$ which according to Lemma 5.16 can be written in the form

with

$$
z_{\gamma}=z_{\gamma}^{\prime} z_{\gamma}^{\prime \prime} z_{\gamma}^{\prime \prime \prime}
$$

$$
z_{\gamma}{ }^{\prime} \in N\left(k_{s}\right), z_{\gamma}{ }^{\prime \prime} \in X(T)_{*}, z_{\gamma}^{\prime \prime \prime} \in T(\widetilde{O})^{(1)} \text {. }
$$

Moreover,

$$
z^{\prime}=\left(z_{\gamma}{ }^{\prime}\right)_{\gamma \in \Gamma} \in Z^{1}\left(\Gamma, N\left(k_{s}\right)\right) .
$$

One can consider the twisted $k$-group $z^{\prime} T$, and it is well known that the family $z^{\prime \prime \prime}=\left(z_{\gamma}^{\prime \prime \prime}\right)_{\gamma \in \Gamma}$ is in fact an element of $Z^{1}\left(\Gamma, z^{\prime} T(\widetilde{O})^{(1)}\right)$. By Lemma $5.17 H^{1}\left(\Gamma, z^{\prime} T(\widetilde{O})^{(1)}\right)=1$. Choose $x \in T(\widetilde{O})^{(1)}$ such that

$$
x^{-1} z_{\gamma}^{\prime \prime \prime} z_{\gamma}^{\prime \gamma} x\left(z_{\gamma}^{\prime}\right)^{-1}=1 \text {. }
$$

An immediate calculation shows that

$$
x^{-1} z_{\gamma}^{\gamma} x \in X(T)_{*} \rtimes N\left(k_{s}\right) .
$$


By Lemma 5.16(4) it now follows that $[z]$ is in the image of our map

$$
\beta_{N}: H^{1}\left(\Gamma, N\left(k_{s}\left[t^{ \pm 1}\right]\right)\right) \longrightarrow H^{1}(\Gamma, N(\widetilde{K}))
$$

as desired.

5.6. Proof of Injectivity. The proof that the natural map

$$
H^{1}\left(\Gamma, G\left(k_{s}\left[t^{ \pm 1}\right]\right)\right) \rightarrow H^{1}(\Gamma, G(\widetilde{K})) .
$$

is injective is the most delicate part of the argument. For convenience we will divide the reasoning into several steps. By Lemma 5.15 the injectivity result we want to prove can be reformulated as follows:

5.20. Proposition. Let $z_{1}=\left(a_{\sigma}\right)_{\sigma \in \Gamma}$ and $z_{2}=\left(b_{\sigma}\right)_{\sigma \in \Gamma}$ be two cocycles in $Z^{1}\left(\Gamma, N\left(k_{s}\left[t^{ \pm 1}\right]\right)\right)$ whose image in $H^{1}(\Gamma, G(\widetilde{K}))$ coincide. Then $z_{1}$ and $z_{2}$ have the same image in $H^{1}\left(\Gamma, G\left(k_{s}\left[t^{ \pm 1}\right]\right)\right)$.

The rest of this section is devoted to the proof of this result.

5.6.1. Linear and translation parts of a cocycle. We begin with a straightforward application of Lemma 5.16 that will be used in the main proof.

5.21. Lemma. There is a unique decomposition $a_{\sigma}=a_{\sigma}^{\prime}(t) a_{\sigma}^{\prime \prime}$ and $b_{\sigma}=$ $b_{\sigma}^{\prime}(t) b_{\sigma}^{\prime \prime}$ where $a_{\sigma}^{\prime}(t), b_{\sigma}^{\prime}(t) \in X(T)_{*}$ and $a_{\sigma}^{\prime \prime}, b_{\sigma}^{\prime \prime} \in N\left(k_{s}\right)$.

We will call the families $z_{1}^{\prime}=\left(a_{\sigma}^{\prime}(t)\right)$ and $z_{2}^{\prime}=\left(b_{\sigma}^{\prime}(t)\right)$ [resp. $z_{1}^{\prime \prime}=\left(a_{\sigma}^{\prime \prime}\right)$ and $z_{2}^{\prime \prime}=\left(b_{\sigma}^{\prime \prime}\right)$ ] the translation [resp. linear] parts of the cocycles $z_{1}$ and $z_{2}$. Since $X(T)_{*}$ is a normal subgroup of $N\left(k_{s}\left[t^{ \pm 1}\right]\right)$, one can easily check that the $z_{1}^{\prime \prime}$ is a cocycle in $Z^{1}\left(\Gamma, N\left(k_{s}\right)\right)$. If we identify $\left({ }_{z_{1}^{\prime \prime}} T\right)\left(k_{s}\left[t^{ \pm 1}\right]\right)=T\left(k_{s}\left[t^{ \pm 1}\right]\right)$ then one checks that the translation parts $z_{1}^{\prime}$ is a cocycle with coefficients in the twisted tori $z_{1}^{\prime \prime} T$. Similar considerations apply to $z_{2}$.

5.22. Remark. The linear part is constant, in the sense that it takes values in $N\left(k_{s}\right)$. The use of the parameter $t$ within the notation $a_{\sigma}^{\prime}(t)$ of the translation part of $a_{\sigma}$ is used to emphasize that $a_{\sigma}$ is being thought as a morphism from $\operatorname{Spec}\left(k_{s}\left[t^{ \pm 1}\right]\right)$ to $T$. This point will become relevant later on when we show that $z_{1}$ and $z_{2}$ are rationally equivalent.

5.6.2. Equality of generic fibers. The first step of the proof of injectivity is to show that the classes $\left[z_{1}\right]$ and $\left[z_{2}\right]$ coincide generically, i.e. that the images of $\left[z_{1}\right]$ and $\left[z_{2}\right]$ coincide in $H^{1}\left(\Gamma, G\left(k_{s}(t)\right)\right)$. The proof is mainly based on the following theorem of Bruhat-Tits.

5.23. Theorem. (Bruhat-Tits, [BT3, 3.15]) Let $H$ be a reductive algebraic group defined over a field $l$. Then the canonical map $H^{1}(l, H) \rightarrow H^{1}(l((t)), H)$ is injective.

A much easier statement of similar flavour is the following. 
5.24. Lemma. Let $H$ be a reductive algebraic group over a field $l$. Then the following are equivalent:

(1) $H$ is irreducible (resp. anisotropic) over l;

(2) $H$ is irreducible (resp. anisotropic) over $l(t)$;

(3) $H$ is irreducible (resp. anisotropic) over $l((t))$.

Proof. We have only to prove that $i) \Longrightarrow$ iii) in both cases.

Assume first that $H$ is irreducible over $l$. If $H_{l((t))}$ is reducible then it has a parabolic subgroup of type $\mathbf{t}$ different than $H$ (see Remark 3.16), and therefore $\operatorname{Par}_{\mathbf{t}}(H)(l((t)))=\operatorname{Par}_{\mathbf{t}}\left(H_{l((t))}\right)(l((t))) \neq \emptyset$. Since $\operatorname{Par}_{\mathbf{t}}(H)$ is proper, we have $\operatorname{Par}_{\mathbf{t}}(H)(l[[t]]) \neq \emptyset$ by the valuative criterion of properness. But then $\operatorname{Par}_{\mathbf{t}}(H)(l) \neq \emptyset$, which is a contradiction. Thus $H_{l((t))}$ is irreducible.

Now we assume that $H$ is anisotropic, namely that $H$ is irreducible and its radical torus $C$ is anisotropic (Proposition 3.17.2). This same Proposition, together with the first step, reduces the proof to showing that $C_{l((t))}$ is anisotropic. The torus $C$ is the twist of $\mathrm{G}_{m}^{r}$ by a continuous morphism $\phi: \operatorname{Gal}\left(l_{s} / l\right) \rightarrow \mathrm{GL}_{r}(\mathbb{Z})$. To say that $C$ is anisotropic is equivalent to $\left(\mathbb{Z}^{r}\right)^{\phi}=0$. Since the map $\operatorname{Gal}\left(l((t))_{s} / l((t))\right) \rightarrow \operatorname{Gal}\left(l_{s} / l\right)$ is split, it follows that $\left(\mathbb{Z}^{r}\right)^{\phi_{l((t))}}=0$ where $\phi_{l((t))}: \operatorname{Gal}\left(l((t))_{s} / l((t))\right) \rightarrow \operatorname{Gal}\left(l_{s} / l\right) \rightarrow \mathrm{GL}_{r}(\mathbb{Z})$ is the composite map. Thus the torus $C \times{ }_{l} l((t))$ is anisotropic.

By our hypothesis on the good characteristic of the base field $k$ there exists a positive integer $n$ not divisible by $\operatorname{char}(k)$ with the property that $n H^{1}\left(F, X(T)_{*}\right)=0$ for all field extension $F / k$. The trick to establish equality of the generic fibers of $z_{1}$ and $z_{2}$ is to add an independent variable $x$ over $k$, and work over the fields $l=k(x)$ and $l_{s}=k_{s}(x)$.

Let $L=l(t), \widehat{L}=l((t))$ and denote by $\widetilde{L}$ the unramified extension of $\widehat{L}$ having residue field $k_{s}(x) / k(x)=l_{s} / l$.

Consider the $l_{s}$-algebra endomorphism of $l_{s}\left[t^{ \pm 1}\right]$ given by $t \rightarrow x t^{n}$. It gives rise to a commutative diagram of fields extensions

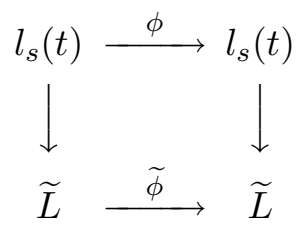

that commutes with the action of the Galois group $\Gamma$. Passing to cohomology we thus obtain the commutative diagram

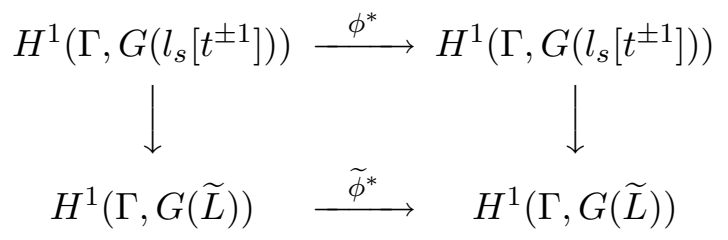

Let $z_{3}=\phi^{*}\left(z_{1}\right)$ and $z_{4}=\phi^{*}\left(z_{2}\right)$. These are cocycles in $Z^{1}\left(\Gamma, N\left(X_{l_{s}}\right)\right)$. Recall that $z_{1}=\left(a_{\sigma}\right), z_{2}=\left(b_{\sigma}\right)$ and that $a_{\sigma}$ and $b_{\sigma}$ can be decomposed into 
their translation and linear parts:

$$
a_{\sigma}=a_{\sigma}^{\prime}(t) a_{\sigma}^{\prime \prime}, \quad b_{\sigma}=b_{\sigma}^{\prime}(t) b_{\sigma}^{\prime \prime}
$$

such that the linear parts are constant, and the translation parts live in $X(T)_{*} \subset T\left(k_{s}\left[t^{ \pm 1}\right]\right)$ (see Remark 5.22). It follows that

$$
\phi^{*}\left(a_{\sigma}\right)=a_{\sigma}^{\prime}\left(x t^{n}\right) a_{\sigma}^{\prime \prime}, \phi^{*}\left(b_{\sigma}\right)=b_{\sigma}^{\prime}\left(x t^{n}\right) b_{\sigma}^{\prime \prime} .
$$

From the way the embedding $X(T)_{*} \hookrightarrow T\left(k_{s}\left[t^{ \pm 1}\right]\right)$ is defined it easily follows that

$$
\begin{gathered}
a_{\sigma}^{\prime}\left(x t^{n}\right)=a_{\sigma}^{\prime}(x) a_{\sigma}^{\prime}\left(t^{n}\right)=a_{\sigma}^{\prime}(x)\left(a_{\sigma}^{\prime}(t)\right)^{n}, \text { and } \\
b_{\sigma}^{\prime}\left(x t^{n}\right)=b_{\sigma}^{\prime}(x) b_{\sigma}^{\prime}\left(t^{n}\right)=b_{\sigma}^{\prime}(x)\left(b_{\sigma}^{\prime}(t)\right)^{n} .
\end{gathered}
$$

5.29. Lemma. The cocycle $z_{3}=\phi^{*}\left(z_{1}\right)$ is equivalent to $\left(a_{\sigma}^{\prime}(x) a_{\sigma}^{\prime \prime}\right)$. Similarly $z_{4}=\phi^{*}\left(z_{2}\right)$ is equivalent to $\left(b_{\sigma}^{\prime}(x) b_{\sigma}^{\prime \prime}\right)$.

Proof. Since $a_{\sigma}=a_{\sigma}^{\prime}(t) a_{\sigma}^{\prime \prime}$ it is clear that $u=\left(a_{\sigma}^{\prime}(x) a_{\sigma}^{\prime \prime}\right)_{\sigma \in \Gamma}$ is a cocycle in $Z^{1}\left(\Gamma, N\left(X_{l_{s}}\right)\right)$. The statement of the Lemma is equivalent to the fact that the image of $z_{3}$ under the canonical bijection $H^{1}\left(\Gamma, N\left(X_{l_{s}}\right)\right) \rightarrow$ $H^{1}\left(\Gamma,{ }_{u} N\left(X_{l_{s}}\right)\right)$ is trivial. By $(5.26)-(5.28)$ this image is $\left(a_{\sigma}^{\prime}(t)\right)^{n}$, and it takes values in the cocharacter group $X\left({ }_{u} T\right)_{*}$ of the twisted torus ${ }_{u} T$. By our assumption on $n$ all elements of $H^{1}\left(\Gamma, X\left({ }_{u} T\right)_{*}\right)$ have exponent $n$. The result follows.

The lemma shows that $z_{3}$ and $z_{4}$ are equivalent to the cocycles $\left(a_{\sigma}^{\prime}(x) a_{\sigma}^{\prime \prime}\right)$ and $\left(b_{\sigma}^{\prime}(x) b_{\sigma}^{\prime \prime}\right)$ respectively. Since the last two cocycles are constant, that is they belong to the image $\operatorname{Im}\left[Z^{1}\left(\Gamma, G\left(k_{s}(x)\right)\right) \rightarrow Z^{1}\left(\Gamma, G\left(k_{s}(x)\left[(t)^{ \pm 1}\right]\right)\right)\right]$, and since the images of the classes of $z_{3}$ and $z_{4}$ in $H^{1}(\Gamma, G(\widetilde{L})) \subset H^{1}(\widehat{L}, G)$ are equal [because of the commutativity of (5.25)], we can conclude with the aid of Theorem 5.23 that the classes of $\left(a_{\sigma}^{\prime}(x) a_{\sigma}^{\prime \prime}\right)$ and $\left(b_{\sigma}^{\prime}(x) b_{\sigma}^{\prime \prime}\right)$ are in fact equal in $H^{1}\left(\Gamma, G\left(k_{s}(x)\right)\right) \subset H^{1}(l, G)=H^{1}(k(x), G)$. This of course completes the proof that $z_{1}$ and $z_{2}$ are generically equivalent, that is $\left[z_{1}\right]_{K}=\left[z_{2}\right]_{K}$ in $H^{1}(K, G)$, as one can see "by replacing x by t".

For later use let us formulate the following by-product of the above trick of introducing the new variable $x$.

5.30. Corollary. Assume that the twisted $K$-group ${ }_{z_{1}} G_{K}$ is irreducible. Then the twisted $\widehat{K}$-group ${ }_{z_{1}} G_{\widehat{K}}$ is also irreducible.

Proof. We reason by contradiction. For convenience let us denote $z_{1} G_{K}$ by $H$. Consider the commutative diagram

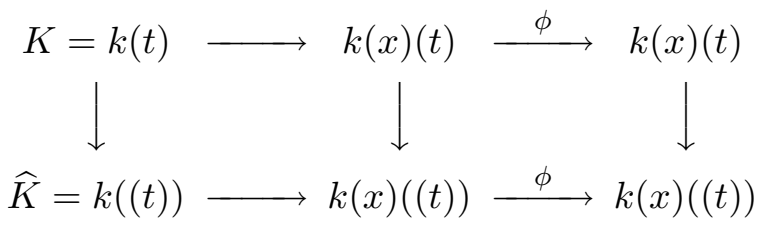


of field extensions. The two maps denoted by $\phi$ are the $k(x)$-morphisms given by $t \rightarrow x t^{n}$, and the rest of the maps are natural inclusions. Assume $H_{\widehat{K}}$ is reducible. By base change along the two bottom arrows we obtain a reducible $k(x)((t))$-group which for convenience we denote by $A$. On the other hand since $H$ is irreducible, by Lemma $5.24 H_{k(t, x)}=H \times_{k(t)} k(t)(x)$ is an irreducible $k(t)(x)=k(x, t)=k(x)(t)$-group. We now apply to $H_{k(t, x)}$ the base change given by $\phi$. The resulting $k(x)(t)$-group, call it $B$, is the twist of $G_{k(x, t)}$ by the cocycle $z_{3}$, which by Lemma 5.29 is a cocycle with values in $G\left(k_{s}(x)\right)$. Since $z_{1}$ becomes $z_{3}$ if we change the variable $t$ to $x$, the twisted group $z_{3} G_{k(x)}$ is irreducible. This implies that $B$ is irreducible. But then $A$, which is obtained form $B$ by the base change $k(x)(t) \rightarrow k(x)((t))$, is according to Lemma 5.24 also irreducible-a contradiction.

5.31. Remark. Let $z \in Z^{1}(X, G)$. If the $X$-group ${ }_{z} G_{X}$ is irreducible then the $K$-group ${ }_{z} G_{K}$ is irreducible. For since the scheme $\mathcal{P}_{\mathbf{t}(P)}$ of parabolic subgroups of ${ }_{z} G_{X}$ of type $\mathbf{t}(P)$ is proper over $X$ and $\operatorname{dim} X=1$, we have $\mathcal{P}_{\mathbf{t}(P)}(K) \neq \emptyset$ if and only if $\mathcal{P}_{\mathbf{t}(P)}(X) \neq \emptyset$. By taking Corollary 5.30 into account we see that if ${ }_{z} G_{X}$ is irreducible then the twisted $\widehat{K}$-group ${ }_{z} G_{\widehat{K}}$ is also irreducible.

5.6.3. Reduction to the irreducible case. The third step of the proof is the reduction to the case when our twisted groups $z_{i} G_{X}$ are irreducible. We begin with a simple result.

5.32. Lemma. Let $G$ be a reductive $k$-group and let $\eta \in Z^{1}(k, G)$ be an arbitrary cocycle. If the injectivity assertion of Theorem 2.4 holds for the twisted k-group ${ }_{\eta} G$ then it holds for $G$.

Proof. Twisting by $\eta$ induces a commutative diagram

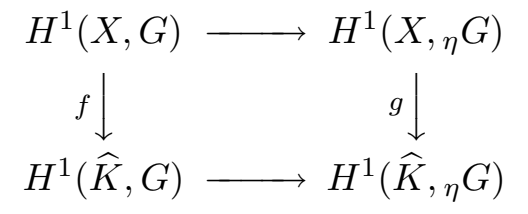

whose horizontal arrows are the twisting bijections. It follows that $f$ is injective if and only if $g$ is injective.

Assume now that our given cocycle $z_{1} \in Z^{1}\left(\Gamma, G\left(k_{s}\left[t^{ \pm 1}\right]\right)\right)$ is such that the twisted $X$-group ${ }_{z_{1}} G_{X}$ is reducible. Let $P_{1}$ be a proper parabolic subgroup of $z_{1} G_{X}$. Choose a $k$-point $x \in X(k)$ and let $\eta=z_{1}(x) \in Z^{1}\left(\Gamma, G\left(k_{s}\right)\right)$ be the fiber of $z_{1}$ at $x$. We set $G^{\prime}={ }_{\eta} G$. Since $G^{\prime}$ is the fiber of ${ }_{z_{1}} G_{X}$ at $x$ we conclude that $G^{\prime}$ contains a parabolic subgroup $P^{\prime}$ of the same type as $P_{1}$.

It follows easily from the definition that if $z_{1}^{\prime}=z_{1} \eta^{-1}$ is the cocycle corresponding to $z_{1}$ under the twisting bijection $H^{1}(X, G) \rightarrow H^{1}\left(X, G^{\prime}\right)$ then the twisted X-groups ${ }_{z_{1}} G_{X}$ and ${ }_{z_{1}^{\prime}} G_{X}^{\prime}$ are isomorphic; in particular $z_{1}^{\prime} G_{X}^{\prime}$ has a parabolic subgroup of the same type as $P^{\prime}$. By taking the last 
Lemma into consideration, and using the fact that the cohomological toral exponent of $G$ and $G^{\prime}$ coincide (Lemma 5.8), we conclude that to establish Proposition 5.20 we may assume without loss of generality that the $k$-group $G$ and the $X$-group $G_{1}={ }_{z} G_{X}$ have parabolic subgroups $P$ and $P_{1}$ of the same type. Under this assumption we then have.

5.33. Lemma. $\left[z_{1}\right] \in \operatorname{Im}\left[H^{1}(X, P) \rightarrow H^{1}(X, G)\right]$.

Proof. Recall the general fact that the isomorphism class of cocycle $z \in$ $Z^{1}(X, G)$ is contained in the image of $H^{1}(X, P)$ if and only if the twisted quotient scheme $z\left(G_{X} / P_{X}\right)$ has an $X$-point. On the other hand by reasoning as in [SGA3, XXIV Prop 4.2.1] we see that ${ }_{z}\left(G_{X} / P_{X}\right)$ is isomorphic to the scheme of parabolic subgroups of type $\mathbf{t}(P)$ of the twisted group scheme ${ }_{z} G_{X}$ (see Remark 3.16). The result follows.

The last Lemma shows that we may assume that $z_{1}$ takes values in $P$. Let $H$ be a Levi subgroup of $P$. Since $X$ is affine, the map $H^{1}(X, H) \rightarrow$ $H^{1}(X, P)$ is bijective [SGA3, XXVI.2.3] so we may additionally assume that $\left[z_{1}\right] \in H^{1}(X, H)$.

We next remark that since $z_{1}$ and $z_{2}$ are rationally equivalent the twisted group scheme $z_{2} G_{K}$ also has a parabolic subgroup of type $\mathbf{t}(P)$. By Remark 5.31 the $X$-group $z_{2} G_{X}$ itself contains a parabolic subgroup of type $\mathbf{t}(P)$. The foregoing reasoning shows that we may also assume that $\left[z_{2}\right] \in Z^{1}(X, H)$.

To complete the reduction to the irreducible case it will suffice to prove that the images of $\left[z_{1}\right]$ and $\left[z_{2}\right]$ under $H^{1}(X, H) \rightarrow H^{1}(\widehat{K}, H)$ are equal (for then if injectivity fails for $G$, it also fails for $H$, and we can assume from the outset that $G$ was chosen of smallest possible dimension so that injectivity fails). For this in turn it suffices to show that the composition (of natural maps)

$$
H^{1}(\widehat{K}, H) \rightarrow H^{1}(\widehat{K}, P) \rightarrow H^{1}(\widehat{K}, G)
$$

is injective. This follows from the following two results.

5.34. Lemma. The mapping $H^{1}(\widehat{K}, H) \rightarrow H^{1}(\widehat{K}, P)$ is bijective.

Proof. This is a special case of [SGA3, XXVI.2.3].

5.35. Lemma. The mapping $H^{1}(\widehat{K}, P) \rightarrow H^{1}(\widehat{K}, G)$ is injective.

Proof. Let $\mu_{1}, \mu_{2} \in Z^{1}(\widehat{K}, P)$ be such that $f\left(\left[\mu_{1}\right]\right)=f\left(\left[\mu_{2}\right]\right)$. After twisting with $\mu_{2}$ we may assume that $\mu_{2}=1$ and $f\left(\left[\mu_{1}\right]\right)=1$. Consider the exact sequence

$$
G(\widehat{K}) \stackrel{g}{\longrightarrow}(G / P)(\widehat{K}) \longrightarrow H^{1}(\widehat{K}, P) \stackrel{f}{\longrightarrow} H^{1}(\widehat{K}, G) .
$$

By Borel-Tits theorem [BT, th. 4.13], the map $g$ is surjective. Hence Ker $f=$ 1 and therefore $\left[\mu_{1}\right]=1$ as desired. One may also quote [SGA3, XXVI.5.10] 
5.6.4. Reduction to anisotropic $k$-loop torsors. Before we can finish the proof of injectivity we need one more result related to a type of torsors, called " $k$ loop torsors" in [GP3], that arise in connections with infinite dimensional Lie theory (see [GP1], [GP2] and [Pi] for details). According to the reformulation given in Proposition 5.20 we may assume that our cocycles $z_{1}$ and $z_{2}$ are of the form $z_{1}=\left(a_{\sigma}\right)_{\sigma \in \Gamma^{\prime}}$ and $z_{2}=\left(b_{\sigma}\right)_{\sigma \in \Gamma^{\prime}}$ where $\Gamma^{\prime}=\operatorname{Gal}\left(k^{\prime} / k\right)$ is the Galois group of a finite extension $k \subset k^{\prime} \subset k_{s}$, and both the $a_{\sigma}$ and the $b_{\sigma}$ belong to $N\left(k^{\prime}\left[t^{ \pm 1}\right]\right)$. After further extending $k^{\prime}$ if necessary, we may further assume that $k^{\prime}$ contains a primitive $n$-root of unity (recall that $n$ is prime to the characteristic of $k$ ) and splits our fixed maximal torus $T \subset G$. Consider a chain of finite field extensions

$$
k(t) \subset k^{\prime}(t) \subset L=k^{\prime}\left(t^{\prime}\right)
$$

where $t^{\prime}=t^{1 / n}$. The extensions $L / k^{\prime}(t)$ and $L / k(t)$ are Galois. Let $\Lambda=$ $\operatorname{Gal}(L / k(t))$ and $\Delta=\operatorname{Gal}\left(L / k^{\prime}(t)\right)$. Clearly we have $\Lambda=\Delta \rtimes \Gamma^{\prime}$ where $\Gamma^{\prime}=\operatorname{Gal}\left(k^{\prime} / k\right)$. The natural mappings $\Lambda \rightarrow \Gamma^{\prime}, G\left(k^{\prime}\right) \rightarrow G\left(k^{\prime}\left(t^{\prime}\right)\right)$ and $G\left(k^{\prime}\left[t^{ \pm 1}\right]\right) \rightarrow G\left(k^{\prime}\left[\left(t^{\prime}\right)^{ \pm 1}\right]\right)$ induce a diagram

$$
\begin{gathered}
H^{1}\left(\Lambda, G\left(k^{\prime}\right)\right) \\
g \downarrow \\
H^{1}\left(\Gamma^{\prime}, G\left(k^{\prime}\left[t^{ \pm 1}\right]\right)\right) \stackrel{f}{\longrightarrow} H^{1}\left(\Lambda, G\left(k^{\prime}\left[\left(t^{\prime}\right)^{ \pm 1}\right]\right)\right.
\end{gathered}
$$

5.36. Lemma. $f\left(\left[z_{1}\right]\right)$ and $f\left(\left[z_{2}\right]\right)$ are in the image of $g$.

Proof. Recall that $z_{1}=\left(a_{\sigma}\right)$ where $\sigma \in \Gamma^{\prime}$ and $a_{\sigma} \in N\left(k^{\prime}\left[t^{ \pm 1}\right]\right) \subset G\left(k^{\prime}\left[t^{ \pm 1}\right]\right)$. Recall also that $a_{\sigma}=a_{\sigma}^{\prime}(t) a_{\sigma}^{\prime \prime}$ where $z_{1}^{\prime}=\left(a_{\sigma}^{\prime}(t)\right)$ and $z_{1}^{\prime \prime}=\left(a_{\sigma}^{\prime \prime}\right)$ are the translation and linear parts of $z_{1}$.

Assume first that the linear part of $z_{1}$ is trivial. Since $\Lambda=\Delta \rtimes \Gamma^{\prime}$, every $\lambda \in \Lambda$ can be written uniquely in the form $\lambda=\delta \sigma$ with $\delta \in \Delta$ and $\sigma \in \Gamma^{\prime}$. With this notation, and according to Remark $5.22, f\left(\left[z_{1}\right]\right)$ is given by a family $\left(a_{\lambda}^{\prime}\left(t^{\prime}\right)\right)$ where

$$
a_{\lambda}^{\prime}\left(t^{\prime}\right)=a_{\sigma}(t)=a_{\sigma}\left(\left(t^{\prime}\right)^{n}\right)=a_{\sigma}\left(t^{\prime}\right)^{n} .
$$

Let $u(t) \in X(T)_{*} \subset T\left(k^{\prime}\left[t^{ \pm 1}\right]\right)$ be such that

$$
a_{\sigma}(t)^{n}=u(t)^{1-\sigma}
$$

We claim that the cocycle $\left(u\left(t^{\prime}\right)^{-1} a_{\lambda}^{\prime}\left(t^{\prime}\right) u\left(t^{\prime}\right)^{\lambda}\right)$ takes values in $G\left(k^{\prime}\right)$. Indeed, let $\lambda=\delta \sigma$. Given that $\delta\left(t^{\prime}\right)=\zeta_{n} t^{\prime}$ where $\zeta_{n}$ is a root of unity of degree $n$ we have

$$
\left(u\left(t^{\prime}\right)\right)^{\delta}=v u\left(t^{\prime}\right)
$$

where $v \in T\left(k^{\prime}\right)$ has entries consisting of roots of unity of degree $n$ [where we identify $T_{k^{\prime}}$ with $\left.\mathrm{G}_{m}{ }^{\mathrm{rk}(G)}\right]$. Furthermore, since $\sigma$ acts trivially on $t^{\prime}$

$$
u\left(t^{\prime}\right)^{\lambda}=\left(u\left(t^{\prime}\right)^{\delta}\right)^{\sigma}=\left(v u\left(t^{\prime}\right)\right)^{\sigma}=v^{\sigma} u\left(t^{\prime}\right)^{\sigma}
$$


and equation (5.37) implies

$$
a_{\sigma}\left(t^{\prime}\right)^{n}=u\left(t^{\prime}\right)^{1-\sigma} .
$$

Thus we have

$$
\begin{gathered}
u\left(t^{\prime}\right)^{-1} a_{\lambda}^{\prime}\left(t^{\prime}\right) u\left(t^{\prime}\right)^{\lambda}=u\left(t^{\prime}\right)^{-1} a_{\sigma}\left(t^{\prime}\right)^{n} u\left(t^{\prime}\right)^{\lambda}= \\
u\left(t^{\prime}\right)^{-1}\left(u\left(t^{\prime}\right) u\left(t^{\prime}\right)^{-\sigma}\right)\left(v^{\sigma} u\left(t^{\prime}\right)^{\sigma}\right)=v^{\sigma}
\end{gathered}
$$

This shows that the cocycle $\left(u\left(t^{\prime}\right)^{-1} a_{\lambda}^{\prime}\left(t^{\prime}\right) u\left(t^{\prime}\right)^{\lambda}\right)$ takes values in $G(k)^{\prime}$ as claimed. Since the class of this cocycle equals $\left.f\left(\left[z_{1}\right]\right)\right]$ the proof of the Lemma in the case when the linear part of $z_{1}$ is trivial is complete.

In general since the linear part is constant, we can consider the twisted $k$-group $z_{1}^{\prime \prime} G$ which for convenience we denote by $G^{\prime}$. We then have the diagram

$$
\begin{gathered}
H^{1}\left(\Lambda, G^{\prime}\left(k^{\prime}\right)\right) \\
g^{\prime} \downarrow \\
H^{1}\left(\Gamma^{\prime}, G^{\prime}\left(k^{\prime}\left[t^{ \pm 1}\right]\right)\right) \stackrel{f^{\prime}}{\longrightarrow} H^{1}\left(\Lambda, G^{\prime}\left(k^{\prime}\left[\left(t^{\prime}\right)^{ \pm 1}\right]\right)\right.
\end{gathered}
$$

One checks that the three relevant twisting bijections are compatible with the maps $f$ and $f^{\prime}$, and $g$ and $g^{\prime}$. This reduces the general problem to the case when the linear part is trivial.

Before concluding the proof of injectivity we need one more final reduction.

5.38. Lemma. Let $C=\operatorname{rad}(G)$ the radical torus of $G$ and consider its maximal split subtorus $C_{d}$. If the natural map $H^{1}\left(X, G / C_{d}\right) \rightarrow H^{1}\left(\widehat{K}, G / C_{d}\right)$ is injective, then the natural map $H^{1}(X, G) \rightarrow H^{1}(\widehat{K}, G)$ is also injective.

Proof. Since $C_{d}$ is central the fibers of the natural map $H^{1}(X, G) \rightarrow H^{1}\left(X, G / C_{d}\right)$ arise as quotients of $H^{1}\left(X, C_{d}\right)=1$, so our map is injective.

5.6.5. Proof of injectivity. We finally come to the proof of Proposition 5.20. By Lemma 5.38 we may assume that the connected centre of our reductive group $G$ is an anisotropic $k$-torus, and that, furthermore, the twisted $X$ groups $z_{1} G_{X}$ and $z_{2} G_{X}$ are irreducible. By Remark 5.31 the $\widehat{K}$-groups $z_{1} G_{\widehat{K}}$ and $z_{2} G_{\widehat{K}}$ are irreducible. Since the radical tori of the $z_{i} G_{\widehat{K}}$ are isomorphic to $\operatorname{rad}(G) \times{ }_{k} \widehat{K}$, it follows from Proposition 3.17.2 that the $\widehat{K}$-groups $z_{1} G_{\widehat{K}}$ and $z_{2} G_{\widehat{K}}$ are anisotropic.

By Lemma 5.36 we may also assume that $z_{1}=\left(a_{\lambda}\right)_{\lambda \in \Lambda}$ and $z_{2}=\left(b_{\lambda}\right)_{\lambda \in \Lambda}$ are cocycles in $Z^{1}\left(\Lambda, G\left(k^{\prime}\right)\right)$ where $\Lambda$ is the Galois group of the extension $L=k^{\prime}\left(t^{\prime}\right)$ of $k(t)$ described in 5.6.4. We will finish the proof by showing that there exists $g \in G\left(k^{\prime}\right)$ such that $a_{\lambda}=g^{-1} b_{\lambda} g^{\lambda}$ for all $\lambda \in \Lambda$.

To this end we consider the two extended Bruhat-Tits buildings [BT2, $\S 4.2 .16] \mathcal{B}_{\widehat{K}}$ and $\mathcal{B}_{\widehat{L}}$ of $G$ over $\widehat{K}$ and $\widehat{L}=k^{\prime}\left(\left(t^{\prime}\right)\right)$ respectively, as well as 
the apartment $\mathcal{A}_{\widehat{L}}$ corresponding to $T_{\widehat{L}}$. Recall that $\mathcal{A}_{\widehat{L}}=X(T)_{*} \otimes_{\mathbb{Z}} \mathbb{R}$ and denote by $o$ its origin. The group $G(\widehat{L})$ acts on $\mathcal{B}_{\widehat{L}}$. We also have a canonical action of $\Lambda=\operatorname{Gal}(L / K) \simeq \operatorname{Gal}(\widehat{L} / \widehat{K})$ on $\mathcal{B}_{\widehat{L}}$ and a canonical identification $\mathcal{B}_{\widehat{K}}=\mathcal{B}_{\widehat{L}}^{\Lambda}$. We also observe that the action of $G(\widehat{L})$ on $\mathcal{B}_{\widehat{L}}$ is $\Lambda$-equivariant. By construction, the point $o$ is fixed by $\Lambda$. Furthermore the $k^{\prime}\left[\left[t^{\prime}\right]\right]$-parahoric group scheme attached to $o$ is nothing but the Chevalley group scheme $G \times{ }_{k} k^{\prime}\left[\left[t^{\prime}\right]\right]$ [BT2, 3.2.13]. Hence the the stabilizer of $o$ in $G(\widehat{L})$ is $G\left(k^{\prime}\left[\left[t^{\prime}\right]\right]\right)$.

The two cocycles $z_{1}$ and $z_{2}$ give rise to two twisted actions of $\Lambda$ on $\mathcal{B}_{\widehat{L}}$, namely $\lambda_{1}(x)=a_{\lambda}(\lambda(x))$ and $\lambda_{2}(x)=b_{\lambda}(\lambda(x))$ for all $x \in \mathcal{B}_{\widehat{L}}$. The invariant subsets in $\mathcal{B}_{\widehat{L}}$ with respect to these two twisted actions of $\Lambda$ are buildings of the twisted $\widehat{K}$-groups ${ }_{z_{1}} G_{\widehat{K}}$ and ${ }_{z_{2}} G_{\widehat{K}}$. Since these twisted $\widehat{K}$-groups are anisotropic, by the Bruhat-Tits-Rousseau's theorem ([Ro] and $[\mathrm{Pr}]$ ) the fixed point sets under consideration consist of unique points. Since $z_{1}$ and $z_{2}$ take values in $G\left(k^{\prime}\right) \subset G\left(k^{\prime}\left[\left[t^{\prime}\right]\right]\right)$ and $G\left(k^{\prime}\left[\left[t^{\prime}\right]\right]\right)$ is the stabilizer of $o$, these fixed points are necessary the origin $o$.

Since $z_{1}$ and $z_{2}$ are cocycles in $Z^{1}\left(\Lambda, G\left(k^{\prime}\right)\right) \subset Z^{1}(\operatorname{Gal}(L / k(t)), G(L))$ which are rationally equivalent, there exists $\widehat{g} \in G(L) \subset G(\widehat{L})$ such that

$$
a_{\lambda}=\widehat{g}^{-1} b_{\lambda} \widehat{g}^{\lambda} \text {. }
$$

It is easy to see that $\widehat{g}^{-1} o$ is invariant with respect to the second twisted action of $\Lambda$. Hence $\widehat{g}^{-1} o=o$, which shows that $\widehat{g} \in G\left(k^{\prime}\left[\left[t^{\prime}\right]\right]\right)$. We now "evaluate (5.39) at $t^{\prime}=0$ ", namely we apply the base change given by the residue map $k^{\prime}\left[\left[t^{\prime}\right]\right] \rightarrow k^{\prime}$. Since this evaluation map commutes with the action of the Galois group $\Lambda$, for $g=\widehat{g}(0) \in G\left(k^{\prime}\right)$ we finally obtain $a_{\lambda}=g^{-1} b_{\lambda} g^{\lambda}$ as desired.

\section{Appendix: The absolutely Reduced subscheme attached to A SCHEME}

Throughout the appendix $k$ denotes a field, $\bar{k}$ an algebraic closure of $k$ and $k_{s}$ the separable closure of $k$ is $\bar{k}$. The nilradical of a (commutative unital) ring $A$ will be denoted by $\mathfrak{n}(A)$. We fix a basis $\left(\alpha_{i}\right)_{i \in I}$ of $\bar{k}$ viewed as a $k$-space. The category of commutative associative and unital algebras over $k$ will be denoted by $k$-alg.

Let $A$ be an object of $k$-alg. For convenience we set $A \otimes_{k} \bar{k}=\bar{A}$. For a subspace $V$ of $A$ we let $\bar{V}=V \otimes_{k} \bar{k} \subset \bar{A}$. We will make repeated use of the following elementary fact.

6.1. Lemma. (Basic Lemma) Let $V$ be a subspace of A. Assume $x=\sum x_{i} \otimes \alpha_{i}$ belongs to $\bar{V} \subset \bar{A}$. Then $x_{i} \in V$ for all $i$.

Proof. Extend a $k$-basis of $V$ to a basis of $A$. 
Following $[\mathrm{RR}]$ we consider the following ideal of $A$ :

$$
\overline{\mathfrak{n}}(A)=\bigcap_{\substack{I \triangleleft A \\ \bar{I} \supset \mathfrak{n}(\bar{A})}} I,
$$

namely the intersection of all ideals $I$ of $A$ for which $\bar{I}=I \otimes_{k} \bar{k}$ contain the nilradical of $\bar{A}$. Note that $\bar{I}=I \bar{A}$.

Recall that given $x \in \bar{A}$ we can uniquely write $x=\sum x_{i} \otimes \alpha_{i}$ where $x_{i} \in A$.

6.2. Lemma. $\overline{\mathfrak{n}}(A)=\left\langle x_{i} \mid x \in \mathfrak{n}(\bar{A})\right\rangle$.

Proof. For convenience let us denote the ideal $\left\langle x_{i} \mid x \in \mathfrak{n}(A)\right\rangle$ by $J$. It is clear from the definition that $\mathfrak{n}(\bar{A}) \subset J \otimes_{k} \bar{k}$. Thus, it will suffices to show that $J \subset I$ whenever $I$ is an ideal of $A$ for which $I \otimes_{k} \bar{k} \supset \mathfrak{n}(\bar{A})$. But this is an immediate consequence of the basic Lemma.

6.3. Remark. It follows from the Lemma (but also from elementary linear algebra considerations) that $J$ does not depend of the choice of basis $\left(\alpha_{i}\right)_{i \in I}$.

6.4. Corollary. For $A$ to be absolutely reduced it is necessary and sufficient that $\overline{\mathfrak{n}}(A)=0$.

6.5. Remark. It is incorrectly asserted in $[\mathrm{RR}]$ that the $\operatorname{ring} A / \overline{\mathfrak{n}}(A)$ is absolutely reduced, namely that $A / \overline{\mathfrak{n}}(A) \otimes_{k} \bar{k}$ is reduced. The following counterexample is due to A. Merkurjev.

6.6. Example. Let $k$ be a separably closed field of characteristic 2 such that $\bar{k} \neq k$. Consider the polynomial $f(x, y)=x^{4}+a y^{2} \in k[x, y]$ where $a \in k$ is not a square. It is easy to see that $f$ is irreducible over $k$ and hence $A=$ $k[x, y] /(f(x, y))$ is a domain. However in $\bar{k}[x, y]$ we have $f(x, y)=\left(x^{2}+b y\right)^{2}$, where $b=\sqrt{a}$, whence $\mathfrak{n}(\bar{A})=\left(\bar{x}^{2}+b \bar{y}\right)$. From Lemma 6.2 it follows that $\overline{\mathfrak{n}}(A)=\left\langle\bar{x}^{2}, \bar{y}\right\rangle$. This implies that the ring

$$
A / \overline{\mathfrak{n}}(A) \simeq k[x, y] /\langle x, y\rangle \simeq k[x] /\left(x^{2}\right)
$$

is not reduced.

6.7. Proposition. Let $\psi: A \rightarrow B$ be a morphism in $k$-alg. If $B$ is absolutely reduced then $\psi$ factors through $A / \overline{\mathfrak{n}}(A)$.

Proof. Since $\mathfrak{n}(\bar{B})=0$ we have $\mathfrak{n}(\bar{A}) \subset \operatorname{ker}(\psi \otimes 1)=\operatorname{ker}(\psi) \otimes \bar{k}$. By taking Lemma 6.2 into consideration, this yields $\overline{\mathfrak{n}}(A) \subset$ ker $\psi$ as desired.

6.8. Lemma. Assume $A$ is a Hopf algebra. Then $\overline{\mathfrak{n}}(A)$ is a Hopf ideal of $A$. 
Proof. Fix a $k$-basis $\left\{a_{s}\right\}_{s \in S}$ of $A$ where $S=S_{1} \sqcup S_{2}$ and $\left\{a_{s}\right\}_{s \in S_{1}}$ is a basis of $\overline{\mathfrak{n}}(A)$. We have the commutative diagram

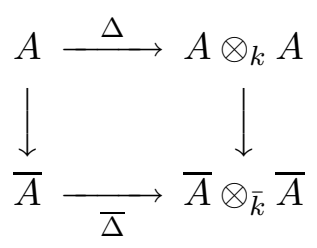

and the canonical isomorphism

$$
\psi: \bar{A} \otimes_{\bar{k}} \bar{A} \rightarrow\left(A \otimes_{k} A\right) \otimes_{k} \bar{k}
$$

Let $x \in \mathfrak{n}(\bar{A})$ and write $x=\sum x_{i} \otimes \alpha_{i}$. By Lemma 6.2 it will suffice to show that

$$
\Delta\left(x_{i}\right) \in \overline{\mathfrak{n}}(A) \otimes A+A \otimes \overline{\mathfrak{n}}(A)
$$

namely that if we write

$$
\Delta\left(x_{i}\right)=\sum_{s, t \in S} c_{s t}^{(i)} a_{s} \otimes a_{t}
$$

then $c_{s t}^{(i)}=0$ whenever both $s$ and $t$ belong to $S_{2}$.

Recall that by Lemma 6.2 we have $\mathfrak{n}(\bar{A}) \subset \overline{\mathfrak{n}}(A) \otimes_{k} \bar{k}$. Since $\mathfrak{n}(\bar{A})$ is a Hopf ideal of $\bar{A}$

$$
\bar{\Delta}(x) \in \mathfrak{n}(\bar{A}) \otimes_{\bar{k}} \bar{A}+\bar{A} \otimes_{\bar{k}} \mathfrak{n}(\bar{A}) .
$$

Thus

$$
\psi(\bar{\Delta}(x)) \in\left(\overline{\mathfrak{n}}(A) \otimes_{k} A\right) \otimes_{k} \bar{k}+\left(A \otimes_{k} \overline{\mathfrak{n}}(A)\right) \otimes_{k} \bar{k} .
$$

On the other hand

$$
\begin{array}{r}
\psi(\bar{\Delta}(x))=\psi\left(\bar{\Delta}\left(\sum_{i} x_{i} \otimes \alpha_{i}\right)\right) \\
=\sum_{i} \psi\left(\bar{\Delta}\left(x_{i} \otimes \alpha_{i}\right)\right) \\
=\sum_{i}\left(\sum_{s, t} c_{s, t}^{(i)} a_{s} \otimes a_{t}\right) \otimes \alpha_{i} \\
=\sum_{i, s, t} a_{s} \otimes a_{t} \otimes c_{s t}^{(i)} \alpha_{i} .
\end{array}
$$

By (6.9) we have $c_{s, t}^{(i)}=0$ whenever both $s$ and $t$ belong to $S_{2}$.

Define a sequence $j_{0}(A) \subset j_{1}(A) \subset \ldots$ of ideals of $A$ inductively as follows:

$$
\begin{aligned}
& j_{0}(A)=\overline{\mathfrak{n}}(A) \\
& j_{i+1}(A)=\left\{x \in A: x+j_{i}(A) \in \overline{\mathfrak{n}}\left(A / j_{i}(A)\right)\right\} .
\end{aligned}
$$


6.10. Proposition. Let $j(A)=\bigcup_{i>0} j_{i}(A)$.

(i) If $A$ is a Hopf algebra then $j(A)$ is a Hopf ideal of $A$.

(ii) If $A$ is noetherian then $A / j(A)$ is absolutely reduced.

(iii) If $\psi: A \rightarrow B$ is a morphism in $k$-alg and $B$ is absolutely reduced, then $\psi$ factors through $A / j(A)$.

Proof. The first assertion follows by induction on $i$ with the aid of Lemma 6.8. By Corollary 6.4 we see that if $j_{i}(A)=j_{i+1}(A)$ then $A / j_{i}(A)$ is absolutely reduced. This establishes (ii). Finally if $\psi$ is as in (iii), then by Proposition $6.7 \psi$ factors through $A / \overline{\mathfrak{n}}(A)$, and one concludes by the inductive definition of $j(A)$.

We assume for the remainder of this section that the $k$-algebra $A$ is of finite type, and denote $A / j(A)$ by $A_{r}$. If $k^{\prime} / k$ is a field extension we have a natural inclusion $\overline{\mathfrak{n}}\left(A \otimes_{k} k^{\prime}\right) \subset \overline{\mathfrak{n}}(A) \otimes_{k} k^{\prime}$. This yields $j\left(A \otimes_{k} k^{\prime}\right) \subset j(A) \otimes_{k} k^{\prime}$, hence a canonical surjective $k^{\prime}$-algebra homomorphism

$$
\chi_{k^{\prime}}:\left(A \otimes_{k} k^{\prime}\right)_{r} \longrightarrow A_{r} \otimes_{k} k^{\prime}
$$

It will be convenient to reformulate the result under consideration in terms of $k$-schemes. Set $X=\operatorname{Spec}(A)$ and $X_{r}=\operatorname{Spec}\left(A_{r}\right)$. Then $\chi_{k^{\prime}}$ corresponds to the closed immersion

$$
\operatorname{Spec}\left(\chi_{k^{\prime}}\right): \operatorname{Spec}\left(A_{r} \otimes_{k} k^{\prime}\right) \longrightarrow \operatorname{Spec}\left(\left(A \otimes_{k} k^{\prime}\right)_{r}\right) .
$$

6.12. Proposition. If $k^{\prime} \subset k_{s}$ then $\chi_{k^{\prime}}$ is an isomorphism.

Proof. We first prove the useful

6.13. Lemma. Let $\overline{X\left(k_{s}\right)}$ denote the closure of $X\left(k_{s}\right) \subset X(\bar{k})$ with respect to the Zariski topology of $X(\bar{k})$. Then $X_{r}(\bar{k})=\overline{X\left(k_{s}\right)}$.

Proof. Since $X_{r}$ is absolutely reduced the inclusion $\overline{X_{r}\left(k_{s}\right)} \subset X_{r}(\bar{k})$ is an equality [Bor, AG 13.3]. Thus

$$
X_{r}(\bar{k})=\overline{X_{r}\left(k_{s}\right)} \subset \overline{X\left(k_{s}\right)}
$$

For the reverse inclusion it will suffice to show that $X\left(k_{s}\right) \subset X_{r}(\bar{k})$ since $X_{r}(\bar{k})$ is a closed subset of $X(\bar{k})$. Now $X\left(k_{s}\right)=\operatorname{Hom}\left(\operatorname{Spec}\left(k_{s}\right), X\right)$. Since $\operatorname{Spec}\left(k_{s}\right)$ is absolutely irreducible any morphism $\phi: \operatorname{Spec}\left(k_{s}\right) \rightarrow X$ factors through $X_{r}$. Thus the inclusion $X_{r}\left(k_{s}\right) \subset X\left(k_{s}\right)$ is in fact an equality.

We now finish the proof of the Proposition. Assume that $k^{\prime} \subset k_{s}$. Then to show that $\chi_{k^{\prime}}$ is an isomorphism we may pass to $\bar{k}$. This corresponds to the closed immersion

$$
\operatorname{Spec}\left(A_{r} \otimes_{k} \bar{k}\right) \hookrightarrow \operatorname{Spec}\left(\left(A \otimes_{k} k^{\prime}\right)_{r} \otimes_{k^{\prime}} \bar{k}\right) .
$$

By the last Lemma both of these affine varieties have the same $\bar{k}$-points, so their underlying topological subspaces agree ([DG] I $\S 3$ prop. 6.8). This means that $A_{r} \otimes_{k} \bar{k}$ is defined by an ideal inside the nilradical of ( $A \otimes_{k}$ $\left.k^{\prime}\right)_{r} \otimes_{k^{\prime}} \bar{k}$. Since this last $\bar{k}$-algebra is reduced (Proposition 6.10 ) the result follows. 
A more functorial approach to the type of problem we have considered in this Appendix can be found in B. Conrad's recent preprint [Crd].

\section{REFERENCES}

[A] P. Abramenko, Group actions on twin buildings, Bull. Belg. Math. Soc. Simon Stevin 3 (1996), 391-406.

[BLR] S. Bosch, W. Lütkebohmert, M. Raynaud, Néron models, Ergebnisse der Mathematik und ihrer Grenzgebiete 21 (1990), Springer-Verlag.

[Bor] A. Borel, Linear Algebraic Groups (Second enlarged edition), Graduate text in Mathematics 126 (1991), Springer.

[BT] A. Borel and J. Tits, Groupes réductifs, Inst. Hautes Études Sci. Publ. Math. 27 (1965), 55-150.

[BT1] F. Bruhat, J. Tits, Groupes réductifs sur un corps local. I. Données radicielles valuées, Inst. Hautes Etudes Sci. Publ. Math. 41 (1972), 5-251.

[BT2] F. Bruhat, J. Tits, Groupes réductifs sur un corps local. II. Schémas en groupes. Existence d'une donnée radicielle valuée, Inst. Hautes Etudes Sci. Publ. Math. 60 (1984), 197-376.

[BT3] F. Bruhat, J. Tits, Groupes réductifs sur un corps local. III. Comléments et applications à la cohomologie galoisienne, J. Fac. Sci. Univ. Tokyo 34 (1987), 671-698.

[Chr] V. Chernousov, The kernel of the Rost invariant, Serre's conjecture II and the Hasse Principle for quasi-split groups ${ }^{3,6} D_{4}, E_{6}$, and $E_{7}$, Math Annalen 326 (2003), 297-330.

[CGR] V. Chernousov, P. Gille, Z. Reichstein, Resolving G-torsors by abelian base extensions, J. Algebra 296 (2006), 561-581.

[CGR2] V. Chernousov, P. Gille, Z. Reichstein, Resolving G-torsors by abelian base extensions II, Manuscripta Mathematica 126 (2008) 465-480.

[CTS1] J.-L. Colliot-Thélène and J.-J. Sansuc, Principal homogeneous spaces under flasque tori: applications, J. Algebra 106 (1987), 148-205.

[CTS2] J.-L. Colliot-Thélène and J.-J. Sansuc, La R-equivalence sur les tores, Annales de l'École Normale Supérieure 10 (1977), 175-229.

[Crd] B. Conrad, Finiteness of class numbers for algebraic groups, preprint.

[DG] M. Demazure, P. Gabriel, Groupes algébriques, North-Holland (1970).

[EGA4] A. Grothendieck (avec la collaboration de J. Dieudonné), Eléments de Géométrie Algébrique IV, Inst. Hautes Etudes Sci. Publ. Math. no 20, 24, 28 and 32 (1964 - 1967).

[G1] P. Gille, La R-équivalence sur les groupes algébriques réductifs définis sur un corps global Inst. Hautes Etudes Sci. Publ. Math. 86 (1997), 199-235.

[G2] P. Gille, Torsors on the affine line, Transformation groups 7 (2002), 231-245.

[G3] P. Gille, Unipotent subgroups of reductive groups in characteristic $p>0$, Duke Math. J. 114 (2002), 307-328.

[GP1] Gille, P. and Pianzola, A. Galois cohomology and forms of algebras over Laurent polynomial rings, Math. Annalen 338 (2007) 497-543.

[GP2] P. Gille and A. Pianzola, Isotriviality and étale cohomology of Laurent polynomial rings, Jour. Pure Applied Algebra, 212 780-800 (2008).

[GP3] P. Gille and A. Pianzola, Galois cohomology and forms of algebras over Laurent polynomial rings $I I$, in progress.

[GS] P. Gille and T. Szamuely, Lectures on the Merkurjev-Suslin's theorem, Cambridge Studies in Advanced Mathematics 101 (2006), Cambridge University Press.

[Gir] J. Giraud, Cohomologie non-abélienne, Springer (1970). 
[Gr] A. Grothendieck, Techniques de descente et théorèmes d'existence en géométrie algébrique. IV. Les schémas de Hilbert, Séminaire Bourbaki, Vol. 6, Exp. No. 221, 249-276, Soc. Math. France, Paris, 1995.

[H1] G. Harder, Halbeinfache Gruppenschemata über Dedekindringen, Invent. Math. 4 (1967), 165-191.

[H2] G. Harder, Halbeinfache Gruppenschemata über vollständigen Kurven, Invent. Math. 6 (1968), 107-149.

[H3] G. Harder, Über die Galoiskohomologie halbeinfacher Matrizengruppen I, Math. Z. 90 (1965), 404-428.

[Kn] M. Knebusch, Grothendieck und Wittringe von nichtausgearteten symmetrischen Bilinearformen, S.-B. Heidelberger Akad. Wiss. Math.-Natur. Kl. 1969/70, 93157.

[K] M. A. Knus, Quadratic and hermitian forms over rings, Grundlehren der mat. Wissenschaften 294 (1991), Springer.

[MPW] A. Merkurjev, I. A. Panin and A. R. Wadworth, Index reduction formulas for twisted flag varieties I, K-Theory 10 (1996) 517-596

[M] J. S. Milne, Étale Cohomology, Princeton University Press.

[Mn] J. Milnor, Algebraic K-theory and quadratic forms, Inventiones Math. 9, 318-344.

[Pi] A. Pianzola, Vanishing of $H^{1}$ for Dedekind rings and applications to loop algebras, C. R. Acad. Sci. Paris, Ser. I 340 (2005), 633-638.

[PR] V. Platonov, A. Rapinchuk, Algebraic Groups and Number Theory, Academic Press, 1994.

[Pr] G. Prasad, Galois-fixed points in the Bruhat-Tits building of a reductive group, Bull. Soc. Math. France 129, (2001), 169-174.

[RR] M. S. Raghunathan, A. Ramanathan, Principal bundles on the affine line, Proc. Indian Acad. Sci. Math. Sci. 93 (1984), 137-145.

[Ro] G. Rousseau, Immeubles des groupes réductifs sur les coprs locaux, Thèse, Université de Paris-Sud (1977).

[Sa] J.-J. Sansuc, Groupe de Brauer et arithmétique des groupes algébriques linéaires sur un corps de nombres, J. Reine Angew. Math. 327 (1981), 12-88.

[SGA1] Séminaire de Géométrie algébrique de l'I.H.E.S., Revêtements étales et groupe fondamental, dirigé par A. Grothendieck, Lecture Notes in Math. 224. Springer (1971).

[SGA3] Séminaire de Géométrie algébrique de l'I.H.E.S., 1963-1964, schémas en groupes, dirigé par M. Demazure et A. Grothendieck, Lecture Notes in Math. 151-153. Springer (1970).

[Se1] J.-P. Serre, Galois Cohomology, Springer, 1997.

[Se2] J.-P. Serre, Cohomologie galoisienne: progrès et problèmes, in "Séminaire Bourbaki, Volume 1993/94, Exposés 775-789", Astérisque 227 (1995), 229-257.

[So] C. Soulé, Chevalley groups over polynomial rings, Homological group theory (Proc. Sympos., Durham, 1977), pp. 359-367, London Math. Soc. Lecture Note Ser. 36 (1979), Cambridge Univ. Press.

[St] R. Steinberg, Regular elements of semisimple algebraic groups Inst. Hautes Etudes Sci. Publ. Math. 25 (1965) 281-312.

[Ti1] J. Tits, Classification of algebraic semisimple groups, in "Algebraic Groups and discontinuous Subgroups" (eds. A. Borel and G. Mostow), Proc. Symp. Pure Math., 9 (1966), 33-62.

[Ti2] J. Tits, Normalisateurs de tores. I. Groupes de Coxeter étendus, J. Algebra 4 (1966), 96-116.

[Ti3] J. Tits, Twin buildings and groups of Kac-Moody type, Groups, combinatorics and geometry (Durham, 1990), 249-286, London Math. Soc. Lecture Note Ser. 165 (1992), Cambridge Univ. Press. 
[Ti4] J. Tits, Résumé de cours au Collège de France 1991-92, Annuaire du Collège de France.

Department of Mathematics, University of Alberta, Edmonton, Alberta T6G 2G1, CANADA

E-mail address: chernous@math.ualberta.ca

UMR 8553 du CNRS, École Normale Supérieure, 45 rue d’Ulm, 75005 Paris, FRANCE.

E-mail address: gille@ens.fr

Department of Mathematics, University of Alberta, Edmonton, Alberta T6G 2G1, CANADA

E-mail address: a.pianzola@math.ualberta.ca 